Part of Journal of Research of the National Bureau of Standards, Volume 24, June 1940

\title{
THEORY OF A SINGLE-LAYER, BIFILAR, ABSOLUTE STANDARD OF MUTUAL INDUCTANCE
}

\author{
By Chester Snow
}

ABSTRACT

A formula is derived for the mutual inductance of two helical wires wound bifilarly upon the same cylindrical form. This aims at a precision sufficient for use in absolute electrical measurements, so that terms are retained which, compared with the principal term, are relatively small quantities of the order of the square of the ratio of pitch to cylindrical radius. Nominally the two helical windings consist of wires of equal diameters, equally spaced, with equal cylindrical radii, but allowance is made for the case where these three equalities are not quite attained. The axial lengths are assumed to be equal, so that, since their pitch must be the same, the total number of turns is the same. The latter is considered to be integral, which permits of the two return currents being carried each by a straight wire parallel to a generator of the cylindrical form and close to it. The effect of these lead wires is included in the formula. The azimuth angle between these generators is assumed to lie between $-\pi$ and $\pi$, so that the point of current entrance for the primary is on a turn adjacent to that of the secondary. Allowance is made for a general type of current distribution.

\section{CONTENTS}

Introduction

II. Mutual inductance, $M$, of the wires as an integral over their sections.- 598

III. Mutual inductance, $m$, of two elementary helical filaments . . . . 603

1. Formula for $m$ in terms of the $\omega$-functions .

2. Principal term, $\omega_{\theta}(l) \ldots$

3. Small term, $\omega_{\theta}(\lambda)$

4. Axial-current term, $\omega_{x}(l)$

5. Azimuth term, $\omega_{a}, \omega_{x}(l)$

6. Second-order approximation for $m_{\ldots}$

IV. Mutual inductance, $M$, of the helical wires... 624

V. Effect of lead wires

VI. Modification required when the reference sections are not quite circular_ 630

VII. Summary

VIII. List of symbols

\section{INTRODUCTION}

Mutual inductors are now being constructed as single-layer, bifilar windings on a circular, cylindrical form. The dimensions may be measured as precisely as those of a self inductor. However, a formula for computing the mutual inductance between bifilar windings is not available. The object of this paper is to supply that need. 


\section{MUTUAL INDUCTANCE, $M$, OF THE WIRES AS AN INTEGRAL OVER THEIR SECTIONS}

The helical wires, $w_{1}$ and $w_{2}$, have the pitch $2 \pi p$. The two full circles of figure 1 with diameters $d_{1}$ and $d_{2}$ are their generating circles, for these circles sweep through the volume of the wires when the plane of the paper is given an angular velocity, $d \theta / d t$, about the $x$ axis, together with a translational velocity, $p d \theta / d t$, in the $x$-direction. The same definition applies to a helical wire with any generating section, such as an ellipse or rectangle. The modifications necessary to extend the formulas to wires with general types of cross section will be indi-

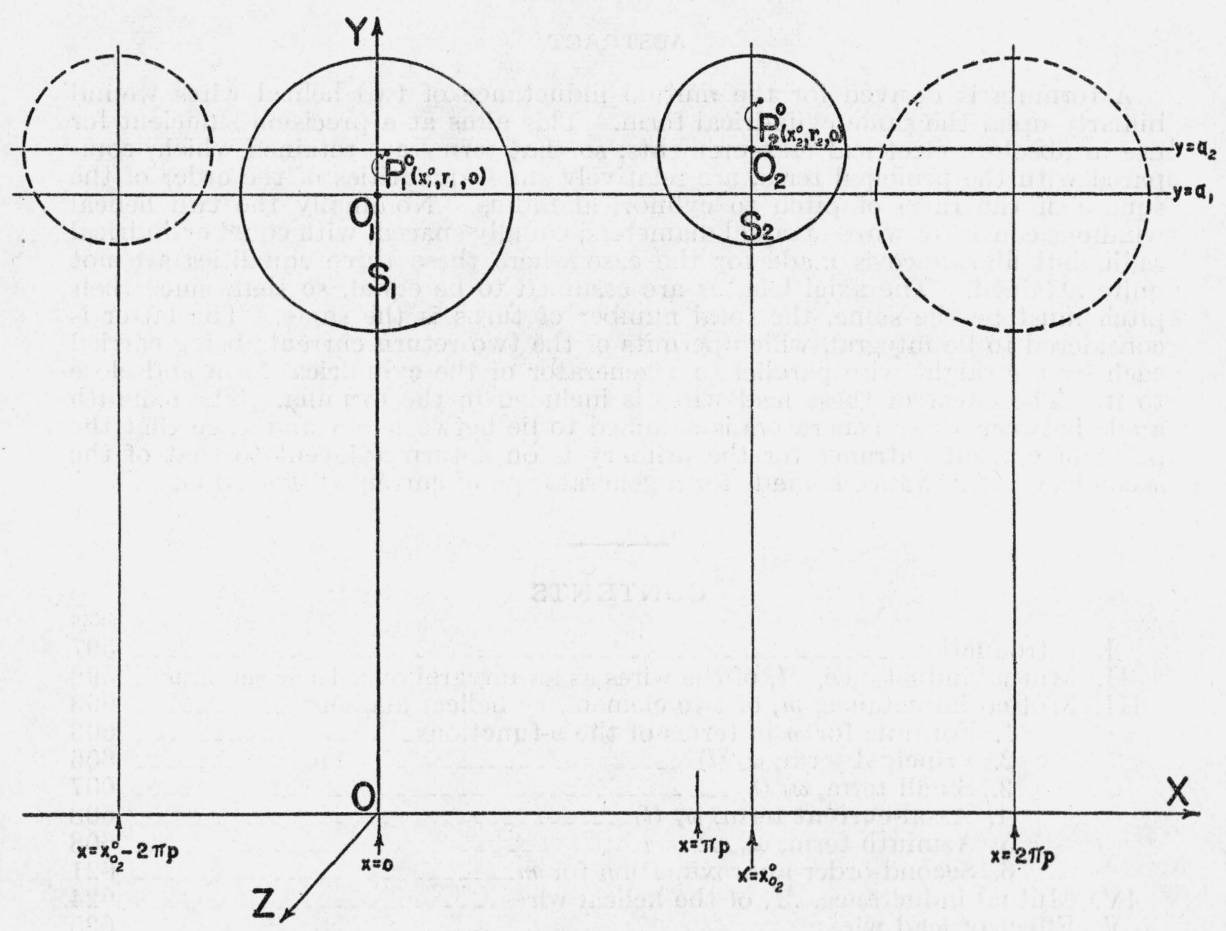

FIGURE 1.--Reference circles which generate the two helical wires.

cated later. The center of the first circle is at $x=0, y=a_{1}, z=0$, that of the second at $x=x_{0_{2}}^{0}, y=a_{2}, z=0$. In the actual construction, the attempt will be made to have the two wires equally spaced $\left(x_{0_{2}}^{0}=\pi p\right)$ and to give them the same mean cylindrical radii $\left(a_{1}=a_{2}\right)$. As neither of these conditions may be attainable with sufficient precision, we consider the more general $x_{0_{2}}^{0}$, and assume $a_{1}-a_{2}$ small compared to the pitch. The two adjacent circular sections bounded by full circles in figure 1 may be designated as the "reference sections" of the wires, to distinguish them from the initial or end sections, where the current enters or leaves the wires. However, the first of these sections, $S_{1}$, may be considered the initial section, $S_{i_{1}}$, of wire $w_{1}$, but the second, $S_{2}$, is in general not the initial section, $S_{i_{2}}$, of wire $w_{2}$. The initial 
section, $S_{t_{2}}$, is the circular section in which $w_{2}$ is cut by a plane which passes through the $x$ axis and makes a given angle, $\alpha$, with the plane $X O Y$, where $-\pi \leqslant \alpha<\pi$.

If $x, y, z$ are rectangular coordinates and $x, r, \theta$ the cylindrical coordinates with the axis, as shown in figure 1 , then the azimuth of the initial section, $S_{i_{1}}$, of $w_{1}$ is $\theta=\theta_{i_{1}}=0$, and that of the end section, $S_{e_{1}}$, is $\theta=\theta_{e_{1}}=2 \pi N$, where $N$ is a (large) given positive integer, the total number of complete turns. The initial and end sections thus being in the same plane, the lead wires for the return current may be made to consist mainly of a straight wire parallel to a generator of the cylindrical form and close to it. Similarly, for wire $w_{2}$ the initial azimuth is $\theta=\theta_{i_{2}}=\alpha$, and the end azimuth is $\theta_{e_{2}}=\alpha+2 \pi N$; so a similar lead wire in the plane $\theta=\alpha$ may be used. These are shown, together with the central helical filaments of $w_{1}$ and $w_{2}$, in figure 2, which is drawn for the case where the wires are equally spaced $\left(x_{0_{2}}^{0}=\pi p\right)$, and where the azimuthal angle is $\alpha=-\pi$ (as explained below).

To designate any particular helix, $h_{1}$, of the helical wire, $w_{1}$, it suffices to assign the two coordinates, $x_{1}^{0}$ and $r_{1}$, of the point $P_{1}^{0}$ in the reference plane of figure 1, through which this helix passes. Then, if $x_{1}, y_{1}, z_{1}$ are the rectangular coordinates of any point $P_{1}$ on this helix, the equations of the helix, $h_{1}$, are

$$
\left.\begin{array}{l}
x_{1}=x_{1}^{0}+p \theta_{1} \\
y_{1}=r_{1} \cos \theta_{1} \\
z_{1}=r_{1} \sin \theta_{1}
\end{array}\right\} \text {, where } 0 \leqslant \theta_{1} \leqslant 2 \pi N \text {. }
$$

It is understood that $x_{1}^{0}$ and $r_{1}$ are constant (as well as $p$ ), and that as the line parameter, $\theta_{1}$, ranges over the prescribed interval, the point $P_{1}$ moves along the helix. The pair of constants $\left(x_{1}^{0}, r_{1}\right)$ may have any values subject to the limitation

$$
x_{1}^{0^{2}}+\left(r_{1}-a_{1}\right)^{2} \leq\left(\frac{d_{1}}{2}\right)^{2} .
$$

The particular pair, $x_{1}^{0}=0, r_{1}=a_{1}$, corresponds to the central helix of wire $w_{1}$. Its equations are

$$
\left.\begin{array}{l}
x_{1}=p \theta_{1} \\
y_{1}=a_{1} \cos \theta_{1} \\
z_{1}=a_{1} \sin \theta_{1}
\end{array}\right\}, \text { where } 0 \leqslant \theta_{1} \leqslant 2 \pi N .
$$

Similarly, any helix, $h_{2}$, of wire $w_{2}$, may be specified by assigning the coordinates of $x_{2}^{0}$ and $r_{2}$ of the point $P_{2}^{0}$ in the reference plane of figure 1 , through which this helix passes or would pass if continued outside the range which limits the line parameter, $\theta_{2}$. The equations of $h_{2}$ are

$$
\left.\begin{array}{l}
x_{2}=x_{2}^{0}+p \theta_{2}=x_{i_{2}}+p\left(\theta_{2}-\alpha\right) \\
y_{2}=r_{2} \cos \theta_{2} \\
z_{2}=r_{2} \sin \theta_{2}
\end{array}\right\} \text {, where } \leqslant \theta_{2} \leqslant \alpha+2 \pi N .
$$

This helix passes through the point in the initial section, $S_{i_{2}}$, whose cylindrical coordinates are $\left(x_{i_{2}}, r_{2}, \alpha\right)$, where $x_{2}^{0}=x_{i_{2}}-p \alpha$. The pair 
of constants $\left(x_{2}^{0}, r_{2}\right)$ which characterize $h_{2}$ are subject to the inequality

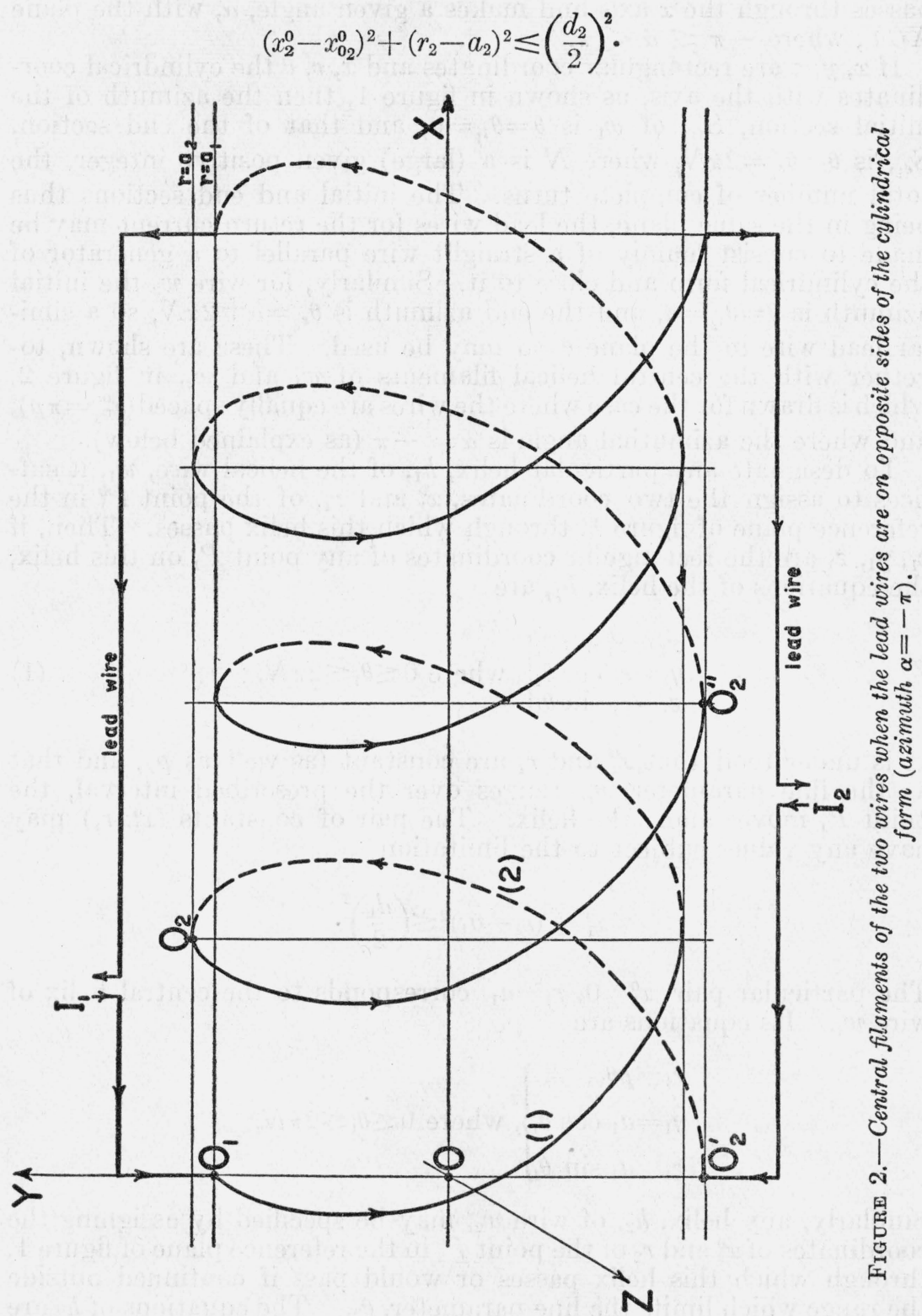

The particular pair, $x_{2}^{0}=x_{0_{2}}^{0}$ and $r_{2}=a_{2}$, correspond to the central helix whose equations are

$$
\left.\begin{array}{r}
x_{2}=x_{0_{2}}^{0}+p \theta_{2} \\
y_{2}=a_{2} \cos \theta_{2} \\
z_{2}=a_{2} \sin \theta_{2}
\end{array}\right\} \text {, where } \alpha \leqslant \theta_{2} \leqslant \alpha+\pi N .
$$


In the special case where the helices are equally spaced, $\left(x_{0_{2}}^{0}=\pi p\right)$, the central helix passes through the point in its initial section, $S_{i_{2}}$, whose cylindrical coordinates are $\left(p(\pi+\alpha), a_{2}, \alpha\right)$ which, for the case $\alpha=-\pi$, becomes $\left(0, a_{2},-\pi\right)$.

This is the point $0_{2}^{\prime}$ in figure 2. The two wires then have the same mean end planes. In the general case, if $-\pi<\alpha<0$, the point $0{ }_{2}^{\prime}$ would be moved toward $0_{2}$ along this helix. If $0<\alpha<\pi$, it would fall between $\mathrm{O}_{2}$ and $0_{2}^{\prime \prime}$.

Every helix in either wire has the same axial length, $l=2 \pi p N$. Every helix, $h_{1}$, in the wire $w_{1}$ has the same initial azimuth, $\theta_{i_{1}}=0$, and end azimuth, $\theta_{e_{1}}=2 \pi N$. They differ in their cylindrical radii, $r_{1}$, and in the initial and end planes. Thus the helix $h_{1}$ lies between the initial plane, $x=x_{l_{1}}=x_{1}^{0}$, and the end plane, $x=x_{e_{1}}=x_{1}^{0}+l$, these limiting planes being 0 and $l$ for the central helix.

Every helix, $h_{2}$, of wire $w_{2}$ has the initial azimuth, $\theta_{t_{2}}=\alpha$, and the end azimuth, $\theta_{e_{2}}=\alpha+2 \pi N$. The helix $h_{2}$ lies between the initial plane, $x=x i_{2}=x_{2}^{0}+p \alpha$, and the end plane, $x=x_{e 2}=x_{l 2}+l$, these limiting planes for the central filament being $x_{02}^{0}$ and $x_{02}^{0}+l$.

It is assumed that $l$ and $a_{1}$ (or $a_{2}$ ) are finite quantities of the same order of magnitude, and that the pitch, $2 \pi p$, is a small quantity of the first order compared with $a_{1}$ or $a_{2}$. A formula for the mutual inductance of the two wires will be obtained which neglects terms that are smaller, relative to the principal part, than the square of this infinitesimal compared with one. It is evident that the diameters of the wires must be small quantities of the same order as the pitch.

In the formula to be obtained, the principal term is proportional to $a_{1} a_{2} / p^{2}$, so that a term proportional to $\sqrt{a_{1} a_{2}} / p$ would be (relatively) a first-order small quantity, the proportionality factors being "finite" functions. Hence a finite function would be relatively of second order. Such terms are not quite negligible, but they need not be evaluated with great precision. In fact the principal term must be of the order of magnitude of $10 \mathrm{mh}\left(10^{7} \mathrm{~cm}\right)$ or larger, if the coil is to be of value in absolute electrical measurements, so that in the "finite", or smallest terms, retained in the formula, an error of $a / 2$ is tolerable. The mutual inductance of a bifilar coil is practically equal to the self inductance of one of the windings. In the case of a coil of 500 turns, $100 \mathrm{~cm}$ long with radius $14 \mathrm{~cm}$, the total self inductance is $17(10)^{6} \mathrm{~cm}$, so that $a / 2$ is $7 \mathrm{~cm}$, or about 4 parts in 10 million. For a shorter coil, $27 \mathrm{~cm}$ long, 135 turns with a larger radius, $22 \mathrm{~cm}$, the self inductance is $7(10)^{6} \mathrm{~cm}$, so that $a / 2=11 \mathrm{~cm}$ is about 4 parts in 3 million. This is the basis for replacing certain expressions defined as infinite series or as definite integrals by simpler expressions in closed form, which are sufficiently close approximations.

Concerning the distribution of the steady current in the wires, we make the assumption that the vector-current density at any point, $P_{1}$, of wire $w_{1}$ has the direction of helix $h_{1}$ passing through that point, and its magnitude remains constant as the point moves along this helix, so that it is some function, $u_{1}\left(x_{1}^{0}, r_{1}\right)=u\left(x_{1}-p \theta_{1}, r_{1}\right)$. The current density will then be a solenoidal vector. Similar assumptions as to the current density in $w_{2}$ make its magnitude some function, $u_{2}\left(x_{2}^{0}, r_{2}\right)$. 
Any axial plane $\left(\theta=\right.$ constant) cuts the wire $w_{1}$ in a circular section of diameter $d_{1}$. If $d S_{1}$ is an element of area of this section, a helical tube of current, whose (oblique) section is $d S_{1}$, carries the current

$$
d I_{1}=\frac{u_{1}\left(x_{1}^{0}, r_{1}\right) d S_{1}}{\sqrt{1+p^{2} / r_{1}^{2}}}
$$

since $\left[1+p^{2} / r_{1}^{2}\right]^{-\frac{1}{2}}$ is the cosine of the angle between the normal to $d S_{1}$ and the direction of flow. The total current carried by the wire is unity, so that

$$
\iint \frac{u_{1}\left(x_{1}^{0}, r_{1}\right) d S_{1}}{\sqrt{1+p^{2} / r_{1}^{2}}}=1
$$

where the integration may be taken over any section of the wire made by the plane $\theta=$ constant.

A precisely similar equation applies to the unit current in wire $w_{2}$. It will be convenient in these integrals, and in those over the same sections which are to be encountered presently, to consider them as taken over the circular reference sections in the plane of figure 1 , even though in the case of the section $S_{2}$, this may not be an actual section of the wire $w_{2}$.

If the wires were of rectangular section, it would be appropriate to take $d S_{1}=d x_{1}^{0} d r_{1}$ and $d S_{2}=d x_{2}^{0} d r$, but since they are circular, it is more natural to use plane polar coordinates $\left(\rho_{1}, \phi_{1}\right)$ for points in $S_{1}$ and $\left(\rho_{2}, \phi_{2}\right)$ for points in $S_{2}$, where

while

$$
x_{1}^{0}=\rho_{1} \cos \phi_{1} \text { and } r_{1}-a_{1}=\rho_{1} \sin \phi_{1}
$$

so that

where

$$
x_{2}^{0}-x_{0_{2}}^{0}=\rho_{2} \cos \phi_{2} \text { and } r_{2}-a_{2}=\rho_{2} \sin \phi_{2} \text {, }
$$

$$
d S_{1}=\rho_{1} d \rho_{1} d \phi_{1} \text { and } d S_{2}=\rho_{2} d \rho_{2} d \phi_{2}
$$

$$
S_{1}=\frac{\pi d_{1}^{2}}{4} \text { and } S_{2}=\frac{\pi d_{2}^{2}}{4}
$$

If $m_{\alpha}\left(x_{1}^{0}, r_{1} ; x_{2}^{0}, r_{2}\right)$ denotes the mutual inductance (Neumann's line integral) of the two helices, $h_{1}$ and $h_{2}$, then the mutual inductance, $M_{\alpha}$, of the two helical wires, $w_{1}$ and $w_{2}$, is

$$
M_{\alpha}=\iint \frac{u_{1}\left(x_{1}^{0}, r_{1}\right) d S_{1}}{\sqrt{1+p^{2} / r_{1}^{2}}} \iint \frac{m_{\alpha}\left(x_{1}^{0}, r_{1} ; x_{2}^{0} r_{2}\right) u_{2}\left(x_{2}^{0}, r_{2}\right)}{\sqrt{1+p^{2} r_{2}^{2}}} d S_{2}
$$

We shall obtain an expression eq 52 for $m_{\alpha}$, whose principal part is constant; so this term contributes its own value to $M_{\alpha}$ when used in eq 4 , as shown by eq 3 for any shape of wire section. The smallest terms of $m$ (second order) may then be evaluated by taking

$$
\frac{u_{1}}{\sqrt{1+p^{2} / r_{1}^{2}}}=\frac{1}{S_{1}} \text { and } \frac{u_{2}}{\sqrt{1+p^{2} / r_{2}^{2}}}=\frac{1}{S_{2}}
$$


where these terms are concerned in the integration of eq 4 . This is also valid for any shape of the sections. For the small terms of the first order we use (when the sections are circular) the Taylor expansions

$$
\begin{aligned}
& \frac{u_{1}\left(x_{1}^{0}, r_{1}\right)}{\sqrt{1+p^{2} / r_{1}^{2}}}=\frac{1}{S_{1}}\left\{1+c_{x_{1}} \rho_{1} \cos \phi_{1}+c_{r_{1}} \rho_{1} \sin \phi_{1}\right\} \\
& \frac{u_{2}\left(x_{2}^{0}, r_{2}\right)}{\sqrt{1+p^{2} / r_{2}^{2}}}=\frac{1}{S_{2}}\left\{1+c_{x_{2}} \rho_{2} \cos \phi_{2}+c_{r_{2}} \rho_{2} \sin \phi_{2}\right\}
\end{aligned}
$$

where the constants $c_{x_{1}}$ and $c_{r_{1}}$ are the values, at the central filament of

$$
c_{x_{1}}=\frac{1}{u_{1}} D_{x_{1}} u_{1} \text { and } c_{r_{1}}=\frac{1}{u_{1}} D_{r_{1}} u_{1}
$$

with similar expressions for $c_{x_{2}}$ and $c_{\tau_{2}}$. In the case of most practical importance, the two wires will be of the same diameter and will have received the same treatment, so that $c_{x_{1}}=c_{x_{2}} \equiv c_{x}$ and $c_{r_{1}}=c_{r_{2}} \equiv c_{r}$. In this case it will be found that the variation with $x^{0}$ is unimportant, as the effect in one wire cancels the effect in the other, so that $c_{x}$ need not be known. The radial variation, however, has some terms which cancel and some which add, giving a term of second order involving $c_{r}$. It may be noted that $c_{r}$ is zero if the current is uniformly distributed over the cross section of the wire. In the "natural" distribution where the current density varies inversely as the cylindrical radius (that is inversely as the length of helical filament), the value of $c_{r}$ is $-1 / a$, where $a$ is the radius of the central filament. If the conductivity of the wire were uniform, it can be shown that the distribution would be "natural."

It may be noted that the integrations in eq 4 are taken over the plane reference sections of figure 1 . The equation is valid for wires with the most general type of cross section. If the sections are not circular, the second members of the two eq 5a must be replaced by other first-order approximations with other variables of integration which are more suitable for the particular type of sections considered. These equations are only necessary for use with the small correction terms which are of first order.

We leave eq 4 at this point and, in section III, digress with tedious detail in pursuit of an approximation for $m$ which may be used in the eq 4. The results are collected in eq 53. After that, in section IV, it is relatively easy (with circular sections) to evaluate the fundamental integral given in eq 4.

\section{MUTUAL INDUCTANCE, $m$, OF TWO ELEMENTARY HELICAL FILAMENTS}

\section{FORMULA FOR $m$ IN TERMS OF THE $\omega$-FUNCTIONS}

The general formula for $m$ has been developed in a recent paper, ${ }^{1}$ in which the small correction terms depending upon the azimuthal angle were reduced to a practicable form by limiting the application to cases where the two helices were nowhere close to each other.

$1 \mathrm{C}$. Snow, Mutual inductance and force between coaxial helical wires. J. Research NBS 22, 239 (1939) RP1178. 
These terms require a different treatment in the present problem of the bifilar inductor, but for all other terms we merely quote the results of that paper. For brevity, this will be referred to as paper 1 .

It is first necessary to place here for reference the function of the modulus $k$ denoted by $\phi_{n}(k)$, which is in fact equal to $\pi^{-1} Q_{n-\frac{1}{2}}$ with argument $\left(2-k^{2}\right) / k^{2}$, where $Q$ is Legendre's function of the second kind. Since $0<k<1$, it is defined for $n=0,1,2,3 * * *$ by the hypergeometric function

$$
\phi_{n}(k)=\frac{k^{2 n+1} \Gamma^{2}(n+1 / 2)}{2 \pi \Gamma(2 n+1)} F\left(n+\frac{1}{2}, n+\frac{1}{2}, 2 n+1 ; k^{2}\right)
$$

or in terms of the complementary modulus $k^{\prime}=\left(1-k^{2}\right)^{\frac{1}{3}}$.

$$
\begin{aligned}
\phi_{n}(k)=-\frac{k^{2 n+1}}{\pi}\left\{F\left(n+\frac{1}{2}, n+\frac{1}{2}, 1 ; k^{2}\right) \log k^{\prime}\right. \\
\left.+\frac{1}{\Gamma^{2}\left(n+\frac{1}{2}\right)} \sum_{s=0}^{\infty} k^{\prime 2 s} \frac{\Gamma^{2}\left(s+n+\frac{1}{2}\right)}{\Gamma^{2}(s+1)}\left[\psi\left(s+n+\frac{1}{2}\right)-\psi(s+1)\right]\right\},
\end{aligned}
$$

where $\psi(z)$ is $\Gamma^{\prime}(z) / \Gamma(z)$. The first two functions are given in terms of the complete elliptic integrals

$$
\pi \phi_{0}(k)=k K(k) \text { and } \pi \phi_{1}(k)=\frac{2}{k}[K(k)-E(k)]-k K(k) .
$$

When $k_{x}$ is a function of $x, r_{1}$, and $r_{2}$ given by

then

$$
k_{x}^{2}=\frac{4 r_{1} r_{2}}{x^{2}+\left(r_{1}+r_{2}\right)^{2}} \text { or } k_{x}^{\prime}=\frac{x^{2}+\left(r_{2}-r_{1}\right)^{2}}{4 r_{1} r_{2}+x^{2}+\left(r_{2}-r_{1}\right)^{2}},
$$

$$
\phi_{n}\left(k_{x}\right)=\sqrt{r_{1} r_{2}} \int_{0}^{\infty} e^{-|x| s} J_{n}\left(r_{1} s\right) J_{n}\left(r_{2} s\right) d s,
$$

where $J_{n}$ is Bessel's function.

Also when $k$ is given by eq $9, \phi_{n}(k)$ is a solution of the partial differential equation

$$
\left\{D_{x}^{2}+D_{r_{1}}^{2}+\frac{1-2 \alpha}{r_{1}} D_{r_{1}}+\frac{\alpha^{2}-n^{2}}{r_{1}^{2}}\right\}_{1_{1}^{\alpha-\frac{1}{2}}} \phi_{n}(k)=0,
$$

where $\alpha$ is any constant. The same equation holds when $r_{1}$ is replaced by $r_{2}$.

The function $\phi_{n}(k)$ is the coefficient in the following Fourier's series:

$$
\frac{k}{4 \sqrt{1-k^{2} \cos ^{2} \theta}}=\frac{1}{2} \phi_{0}(k)+\sum_{n=1}^{\infty} \phi_{n}(k) \cos 2 n \theta .
$$

In the following, it will be assumed that $k_{x}$ and $k_{x}^{\prime}$ are the functions of $x, r_{1}$ and $r_{2}$ defined in eq 9 . When $x$ is merely replaced by $x_{1}$, this 
is indicated by $k_{1}$; when $x$ is $l$, by $k_{i}$; and when $x$ is zero, by $k_{0}$; similarly, $k_{\lambda}$, when $x=\lambda$. The fundamental equation is eq 13 of paper 1 .

$$
m=\omega\left(x_{e_{2}}-x_{i_{1}}\right)-\omega\left(x_{e 2}-x_{e_{1}}\right)+\omega\left(x_{i_{2}}-x_{e_{1}}\right)-\omega\left(x_{i_{2}}-x_{i_{1}}\right),
$$

where the subscripts $i$ and $e$ indicate initial and end planes, and $\omega(x)$ is written in place of $\omega\left(x, r_{1}, r_{2} ; \alpha\right)$. This function was there resolved into three constituents, as follows:

$$
\omega(x)=\omega_{\theta}(x)+\omega_{x}(x)+\omega_{a}(x, \alpha) .
$$

The term $\omega_{a}$ was called the azimuthal $\omega$-function, as it is the only one depending upon $\alpha$. All three functions depend upon $r_{1}$ and $r_{2}$.

The associated current sheet of the helix, $h_{1}$, is defined as the circular cylindrical surface coaxial with it, having the same radius, $r_{1}$, and end planes, $x_{i_{1}}, x_{e_{1}}$, on which surface the linear density of current has the angular component, $j_{\theta}=1 / 2 \pi p=n=$ the number of turns per centimeter. The axial component of current is $j_{x}=1 / 2 \pi r_{1}$, so that the unit current in the $x$ direction is uniformly distributed over the sheet. The two terms, $\omega_{\theta}(x)+\omega_{x}(x)$, when used in eq 11 give the mutual inductance of the two current sheets which are associated with the helical filaments, $h_{1}$ and $h_{2}$. The first, $\omega_{\theta}(x)$, is that due to their angular or $\theta$ components of current, and this contributes the principal part to $m$. The second, $\omega_{x}(x)$, is relatively a second-order infinitestimal, representing the effect of the axial components of current. Each of these functions is an even function of its $x$-argument, vanishing with it. This property used in eq 11 gives the following interpretation of these functions.

Let the helices have the same end planes, $x_{i_{1}}=x_{i_{2}}$ and $x_{e_{1}}=x_{e_{2}}$. Then, if $x=x_{i_{1}}-x_{e_{1}}$, the function $2 \omega_{\theta}(x)+2 \omega_{x}(x)$, or explicitly $2 \omega_{\theta}\left(x, r_{1}, r_{2}\right)+2 \omega_{x}\left(x, r_{1}, r_{2}\right)$, represents the mutual inductance of two coaxial circular cylindrical current sheets of axial length $x$ and radii $r_{1}$ and $r_{2}$, when they have the same end planes.

The integral definitions of these functions are

$$
\begin{aligned}
& \omega_{\theta}(x)=\frac{\sqrt{r_{1} r_{2}}}{p^{2}} \int_{0}^{x} d x_{2} \int_{0}^{x_{2}} \phi_{1}\left(k_{1}\right) d x_{1} \\
& \omega_{x}(x)=\frac{1}{\sqrt{r_{1} r_{2}}} \int_{0}^{x} d x_{2} \int_{0}^{x_{2}} \phi_{0}\left(k_{1}\right) d x_{1}
\end{aligned}
$$

The azimuthal function, $\omega_{a}(x, \alpha)$, is written

$$
\omega_{a}(x, \alpha)=\sum_{n=1}^{\infty} \omega_{n}(x, \alpha),
$$

where

$\omega_{n}(x, \alpha)=\frac{\sqrt{r_{1} r_{2}}}{p^{2}} \int_{0}^{x} d x_{2} \int_{0}^{x_{2}}\left[\phi_{n-1}\left(k_{1}\right)+\phi_{n+1}\left(k_{1}\right)\right] \cos \frac{n}{p}\left(x_{1}-x+p \alpha\right) d x_{1}$. 
It will be found that $\omega_{a}$ is also infinitesimal compared with the principal term $\omega_{0}$ but, unlike $\omega_{x}$, it contains first-order terms.

If, for brevity, we define the small quantity $\lambda$ by

$$
\lambda \equiv x_{i_{2}}-x_{i_{1}}=x_{2}^{0}-x_{1}^{0}+p \alpha,
$$

the fundamental eq 11 becomes

$$
\begin{aligned}
& m=\omega_{\theta}(l+\lambda)+\omega_{\theta}(l-\lambda)-2 \omega_{\theta}(\lambda)+2 \omega_{x}(l) \\
& \quad+\omega_{a}(l+\lambda, \alpha)+\omega_{a}(-l+\lambda, \alpha)-2 \omega_{a}(\lambda, \alpha) .
\end{aligned}
$$

Since $\lambda$ is small, this becomes

$$
\begin{aligned}
m & =2 \omega_{\theta}(l)-2 \omega_{\theta}(\lambda)+2 \omega_{x}(l)+4 \sqrt{r_{1} r_{2}}\left(\frac{\lambda}{2 \pi p}\right)^{2} \pi^{2} \phi_{1}\left(k_{l}\right) \\
& +\omega_{a}(l+\lambda, \alpha)+\omega_{a}(-l+\lambda, \alpha)-2 \omega_{a}(\lambda, \alpha) .
\end{aligned}
$$

In the evaluation of these $\omega$-functions which follows, frequent use will be made of the results and methods of an earlier paper, ${ }^{2}$ which will be referred to as paper 2 . Reference will also be made to paper $3 .^{3}$

\section{PRINCIPAL TERM, $\omega_{\theta}(l)$}

The principal term, $2 \omega_{\theta}(l)$, in eq 15 represents the mutual inductance of two coaxial current sheets of radii $r_{1}$ and $r_{2}$, which have common end planes and axial length, $l$. It is due to their angular component of current only. When $r_{2} \rightarrow r_{1}$, this becomes the self inductance of the circular cylindrical sheet.

Since $r_{2}-r_{1}$ is small, it will be appropriate to express this term as a self inductance corresponding to the cylindrical radius $a$, which is the arithmetic mean of the radii $a_{1}$ and $a_{2}$ of the two central filaments.

The self inductance of the current sheet of length, $l$, and this radius $a$ is

$$
L_{s}=\frac{4 \pi N^{2}}{3} \sqrt{l^{2}+D^{2}}\left[K-E+\frac{D^{2}}{l^{2}}(E-k)\right]
$$

where the modulus $k$ is now given by

$$
k^{2}=\frac{D^{2}}{l^{2}+D^{2}} \text { and } D=2 a=a_{1}+a_{2} .
$$

In eq 19 of appendix 3 of paper 2 is given an expansion which, to the second order inclusive, is, by a slight change in notation, equivalent to the following equation:

$$
\begin{aligned}
& 2 \omega_{\theta}(l)=L_{s}-l \frac{\left|r_{2}-r_{1}\right| \sqrt{r_{1} r_{2}}-2 \pi a\left(\frac{r_{2}-r_{1}}{2 \pi p}\right)^{2} \log \left(\frac{r_{2}-r_{1}}{8 a}\right)^{2}}{p^{2}}+32 \pi a\left\{A_{1}\left(\frac{a}{2 \pi p}\right)\left(\frac{r_{1}-a+r_{2}-a}{2 \pi p}\right)\right. \\
& \left.+A_{3}\left(\frac{r_{1}-a}{2 \pi p}\right)\left(\frac{r_{2}-a}{2 \pi p}\right)+A_{4}\left(\frac{r_{2}-r_{1}}{2 \pi p}\right)^{2}\right\},
\end{aligned}
$$

'C. Snow, Formula for the self inductance of a helix made with wire of any section, BS Sci. Pap. 21, 431 (1926-27) S537.

$3 \mathrm{C}$. Snow, $A$ simplified precision formula for the inductance of a helix with corrections for the lead-in wires, BS J. Research 9, 419 (1932) RP479. 
where the constants $A_{1}, A_{3}$, and $A_{4}$ are functions of the modulus, $k$, which was defined in eq 17 , as follows:

$$
\left.\begin{array}{l}
A_{1}=\frac{1}{2}\left[\frac{E}{k}-1\right] \\
A_{3}=\frac{1}{2 k}\left[2 E-\left(1-k^{2}\right) K\right]-1 \\
A_{4}=\frac{1}{8}\left[\frac{4 E-\left(2-k^{2}\right) K}{k}-\frac{5}{2}\right]
\end{array}\right\}
$$

It is evident that in the fourth term of eq 15 we may place $\sqrt{r_{1} r_{3}}=a$ and $\phi_{1}\left(k_{\imath}\right)=\phi_{1}(k)$, since this term is of the smallest order retained (second order).

\section{SMALL TERM, $\omega_{\theta}(\lambda)$}

To simplify the term $-2 \omega_{\theta}(\lambda)$ (on account of the fact that $\lambda$ is small), we write, by eq 15 and $13 \mathrm{a}$,

$$
-2 \omega_{\theta}(\lambda)=-\frac{2 \sqrt{\left(r_{1} r_{2}\right.}}{p^{2}} \int_{0}^{\lambda}\left(\lambda-x_{1}\right) \phi_{1}\left(k_{1}\right) d x_{1} .
$$

But since $\lambda$ is small, $x_{1}$ is small throughout the range of this integration. Also $r_{2}-r_{1}$ is small, so that by eq $9 k_{1}^{\prime}$ is small. Therefore, in this integral we may use eq 7 , which gives

$$
\begin{aligned}
-2 \phi_{1}\left(k_{1}\right) & =\frac{2}{\pi}\left[\log k_{1}^{\prime}+\psi\left(\frac{3}{2}\right)-\psi(1)\right]+\text { infinitesimals } \\
& =\frac{2}{\pi}\left[\log k_{1}^{\prime}+2-2 \log 2\right] \\
& =\frac{1}{\pi}\left\{4-2 \log 8 \sqrt{r_{1} r_{2}}+\log \left[x_{1}^{2}+\left(r_{2}-r_{1}\right)^{2}\right]\right\}
\end{aligned}
$$

Hence $\omega_{\theta}(\lambda)$ is an even function of $\lambda$, which differs infinitesimally from the following integral:

$$
-2 \omega_{\theta}(\lambda)=\frac{\sqrt{r_{1} r_{2}}}{\pi p^{2}} \int_{0}^{\lambda}\left(\lambda-x_{1}\right)\left\{4-2 \log 8 \sqrt{r_{1} r_{2}}+\log \left[x_{1}^{2}+\left(r_{2}-r_{1}\right)^{2}\right]\right\} d x_{1} .
$$

The result of this integration and of others which are to follow may be abbreviated by introducing the complex variables

and

$$
z \equiv i\left[\lambda+i\left(r_{2}-r_{1}\right)\right]=-\left(r_{2}-r_{1}\right)+i\left(x_{2}^{0}-x_{1}^{0}+p \alpha\right) .
$$

$$
z^{\prime} \equiv\left|r_{2}-r_{1}\right|+i|\lambda| \text {. }
$$

Then $\omega_{\theta}(\lambda)$ is a relatively small term of second order, given by the real part of

$$
\begin{gathered}
-2 \omega_{\boldsymbol{\theta}}(\lambda)=\pi a\left\{\left(\frac{\lambda}{2 \pi p}\right)^{2}\left[2-4 \log \frac{8 a}{2 \pi p}\right]+2\left(\frac{r_{2}-r_{1}}{2 \pi p}\right)^{2} \log \left(\frac{r_{2}-r_{1}}{2 \pi p}\right)^{2}\right. \\
\left.-4\left(\frac{z}{2 \pi p}\right)^{2} \log \left(\frac{z}{2 \pi p}\right)\right\}
\end{gathered}
$$




\section{AXIAL-CURRENT TERM, $\omega_{x}(l)$}

This term of second order may be treated as a constant. It may be computed by

$$
2 \omega_{x}(l)=2 l \omega_{x}^{\prime}(l)-\frac{4 D}{\pi}\left(\frac{E}{k}-1\right),
$$

where, by eq 43 of paper 1 ,

$$
\omega_{x}^{\prime}(l)=\log \frac{l+\sqrt{l^{2}+D^{2}}}{D}+\frac{l}{\sqrt{l^{2}+D^{2}}} \log 2 .
$$

This approximation was derived on the assumption (here adopted) that terms in the $\omega$-functions of order of magnitude $.1 D$ are negligible ( 3 parts in $10^{7}$ ).

In paper 3 (eq 8) an approximation was used which is equivalent to

$$
\begin{aligned}
2 \omega_{x}^{\prime}(l)=P\left(\frac{k^{\prime}}{k}\right) & =\frac{k^{2}}{4 k^{\prime 2}}+2 \log \frac{4 k^{\prime}}{k}, \text { if } k^{\prime}>k \\
& =\frac{3 k^{\prime}}{k}-\frac{k^{\prime}}{k} \log \frac{k^{\prime}}{k}, \text { if } k^{\prime}<k .
\end{aligned}
$$

Either eq 23 or $23^{\prime}$ is adequate for computing this relatively small term. The former has the advantage of a single form, and is one which blends naturally with the corresponding terms representing the effect of the lead wires (eq 62, below).

\section{AZIMUTH TERM, $\omega_{a}$}

In paper 1, pages $255-258$, it is shown that instead of the integral $13 \mathrm{~d}$ for $\omega_{n}$ we may take

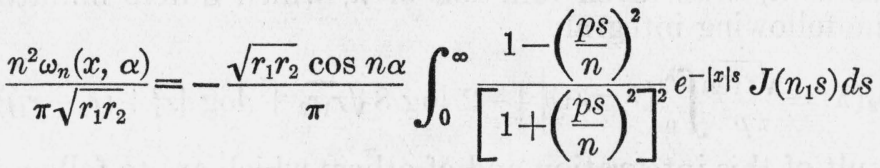

$$
\begin{aligned}
& \pm\left\{\left[\frac{n x}{\pi p} \cos n\left(\alpha-\frac{x}{p}\right)+\left(\frac{1}{\pi}+\frac{n\left|r_{2}-r_{1}\right|}{\pi p}\right) \sin n\left(\alpha-\frac{x}{p}\right)\right] e^{\frac{-n\left|r_{2}-r_{1}\right|}{p}}\right. \\
& \left.-\sin n \alpha \frac{2 p \sqrt{r_{1} r_{2}}}{\pi n} \int_{0}^{\infty} \frac{s e^{-|x| s} J(n, s)}{\left[1+\left(\frac{p s}{n}\right)^{2}\right]^{2}} d s\right\}
\end{aligned}
$$

where for brevity

$$
J(n, s) \equiv J_{n-1}\left(r_{1} s\right) J_{n-1}\left(r_{2} s\right)+J_{n+1}\left(r_{1} s\right) J_{n+1}\left(r_{2} s\right) .
$$

The upper or lower of the ambiguous signs is to be taken according as $x$ is positive or negative, the quantity affected with the \pm sign being 
zero when $x$ is zero. This expression 24 is not equal to the integral definition of $\omega_{n}$ given in eq 13d. It is, however, effectively the same since terms have been discarded which cancel out of eq 11. Such a procedure is allowable if we make eq 24 the basis for simplifying the function $\omega_{n}(k)$ both in the case where $x$ is small and when it is finite, so that the terms in question actually are discarded from each of the four $\omega$-functions occurring in eq 11.

The simplification of eq 24 depends upon the fact that we may neglect all terms in this equation which are infinitesimals of higher order than the second in comparison with $\omega_{\theta}$, which contains the factor $1 / p^{2}$.

When $x$ is finite, the second integral in eq 24 is negligible, and the first may be replaced by

$$
\int_{0}^{\infty} e^{-|x| s} J(n, s) d s=\frac{1}{\sqrt{r_{1} r_{2}}}\left[\phi_{n-1}(k)+\phi_{n+1}(k)\right]_{3}
$$

as shown by eq 10. This is an even function of $x$. Hence

$$
\begin{aligned}
& \omega_{a}(l+\lambda, \alpha)+\omega_{a}(l-\lambda, \alpha)= \\
& =\pi \sqrt{r_{1} r_{2}}\left\{\frac{2 l}{\pi p} \sum_{n=1}^{\infty} \frac{1}{n} e^{\left.\frac{-n\left|r_{-}-r_{1}\right|}{p} \cos \frac{n\left(x_{2}^{0}-x_{1}^{0}\right)}{p}-\frac{2}{\pi} \sum_{n=1}^{\infty}\left[\phi_{n-1}\left(k_{l}\right)+\phi_{n+1}\left(k_{e}\right)\right] \frac{\cos n \alpha}{n^{2}}\right\}}\right. \\
& =-\pi \sqrt{r_{1} r_{2}}\left\{\frac { 2 l } { \pi p } \operatorname { l o g } \left[1-e^{\left.-\left(\frac{\left|r_{2}-r_{1}\right|+i\left(x_{i}^{0}-x_{i}^{0}\right)}{p}\right)\right]}\right.\right. \\
& \left.+\frac{2}{\pi} \sum_{n=1}^{\infty} \frac{\left[\phi_{n-1}\left(k_{l}\right)+\phi_{n+1}\left(k_{l}\right)\right]}{n^{2}} \cos n \alpha\right\}
\end{aligned}
$$

where the real part is to be taken.

The logarithmic term containing the factor $l / p$ is the most important correction term. The series in $\cos n \alpha$ is the smallest order of magnitude retained (second order relative to $\omega_{\theta}$ ). It is shown in eq 57 of paper 1 that an approximation in finite form sufficient in all cases is given by

$$
\begin{array}{r}
\frac{2}{\pi} \sum_{n=1}^{\infty}\left[\frac{\phi_{n-1}(k)+\phi_{n+1}(k)}{n^{2}}\right] \cos n \alpha=\frac{2 \pi}{3} \phi_{1}(k)+\frac{1}{k} \sqrt{1-k^{2} \cos ^{2} \frac{\alpha}{2}} \\
-\left(\frac{k^{1}}{k}\right)^{2}\left(\cos \frac{\alpha}{2}\right) \sin ^{-1}\left(k \cos \frac{\alpha}{2}\right)-\frac{1}{k^{2}} \sin \frac{\alpha}{2} \log \left[\frac{k \sin \frac{\alpha}{2}+\sqrt{1-k^{2} \cos ^{2} \frac{\alpha}{2}}}{k^{\prime}}\right]
\end{array}
$$

(By a misprint the logarithm had the factor $1 / k$ instead of $1 / k^{2}$ in eq 57 , but this was used correctly in the equations depending upon it.) 
The derivation of eq 27 depends upon the fact that the continuous, even function of $\theta, f(\theta)$, which is never negative and is a periodic function of $\theta$ with period $2 \pi$, defined for all real values of $\theta$ by the series

$$
f(\theta)=\frac{2}{3}-\frac{4}{\pi^{2}} \sum_{n=1}^{\infty} \frac{\cos n \theta}{n^{2}}
$$

is represented approximately, for all values of $\theta$, by

$$
f(\theta) \approx\left|\sin \frac{\theta}{2}\right| .
$$

The exact expression for $f(\theta)$ is

$$
f(\theta)=4\left(\frac{\theta}{2 \pi}\right)\left(1-\frac{\theta}{2 \pi}\right) \text { for the range } 0 ₹ \theta ₹ 2 \pi
$$

or

$$
f(\theta)=4\left[\left|\frac{\theta}{2 \pi}\right|-\left(\frac{\theta}{2 \pi}\right)^{2}\right] \text { for the range }-2 \pi<\theta<2 \pi .
$$

The graph of $f(\theta)$ is a continuous curve represented by a succession of parabolic arcs. Its slope is discontinuous at $\theta=0$ or $2 \pi n$.

Using eq 27, we find that expression 26 becomes

$\omega_{a}(l+\lambda, \alpha)+\omega_{a}(l-\lambda, \alpha)$

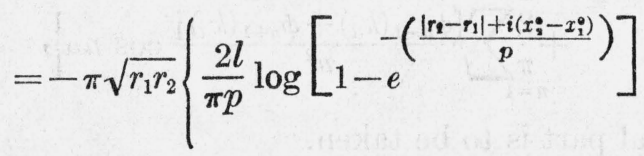

$$
\begin{aligned}
& +\frac{2 \pi}{3} \phi_{1}\left(k_{l}\right)+\frac{1}{k_{l}} \sqrt{1-k_{l}^{2} \cos ^{2} \frac{\alpha}{2}} \\
& -\left(\frac{k_{l}^{\prime}}{k_{l}}\right)^{2} \cdot\left(\cos \frac{\alpha}{2}\right) \sin ^{-1}\left(k_{l} \cos \frac{\alpha}{2}\right) \\
& \left.-\frac{1}{k_{l}^{2}} \sin \frac{\alpha}{2} \cdot \log \left[\frac{k_{l} \sin \frac{\alpha}{2}+\sqrt{1-k_{l}^{2} \cos ^{2} \frac{\alpha}{2}}}{k_{l}^{\prime}}\right]\right\} \text {. }
\end{aligned}
$$

We next consider the last term in eq 15 , which is $-2 \omega_{a}(\lambda, \alpha)$. Since $\lambda$ is small, we must now consider all the terms in eq 24 in order to obtain valid expressions for $\omega_{n}(\lambda, \alpha)$. Considering $\lambda$ as an infinitesimal of the same order as the pitch or smaller, it is evident that the ratio, $\lambda / p$, may be infinitesimal, but in general may have a finite value positive or negative. 
Reference to eq $10 a$ shows that we may write

$$
\begin{aligned}
& -\frac{\sqrt{\left(r_{1} r_{2}\right.}}{\pi} \int_{0}^{\infty} \frac{1-\left(\frac{p s}{n}\right)^{2}}{\left[1+\left(\frac{p s}{n}\right)^{2}\right]^{2}} e^{-|\lambda| s J(n, s) d s=-\frac{1}{\pi}\left[\phi_{n-1}\left(k_{\lambda}\right)+\phi_{n+1}\left(k_{\lambda}\right)\right]} \\
& +\frac{\sqrt{r_{1} r_{2}}}{\pi} \int_{0}^{\infty}\left(\frac{p s}{n}\right)^{2}\left\{\frac{2}{\left[1+\left(\frac{p s}{n}\right)^{2}\right]^{2}}+\frac{1}{1+\left(\frac{p s}{n}\right)^{2}}\right\} e^{-|\lambda| s} J(n, s) d s,(30)
\end{aligned}
$$

where the last integral is no longer infinitesimal with $p$, because $\lambda$ is also infinitesimal. In fact, the substitution $s=n t / p$ gives

$$
\begin{aligned}
&\left.I \equiv \frac{\sqrt{r_{1} r_{2}}}{\pi} \int_{0}^{\infty}\left(\frac{p s}{n}\right)^{2}\left\{\frac{2}{\left[1+\left(\frac{p s}{n}\right)^{2}\right]^{2}}+\frac{1}{1+\left(\frac{p s}{n}\right)^{2}}\right] e^{-|\lambda| s} J n, s\right) d s \\
&=\frac{n \sqrt{r_{1} r_{2}}}{\pi p} \int_{0}^{\infty} t^{2}\left\{\frac{2}{\left[1+t^{2}\right]^{2}}+\frac{1}{1+t^{2}}\right\} e^{-\left|\frac{n}{p}\right| t} J\left(n, \frac{n t}{p}\right) d t,
\end{aligned}
$$

which shows the integral is a function of the finite ratio, $|\lambda / p|$. Referring to eq 25 , we find

$$
J\left(n, \frac{n t}{p}\right)=J_{n-1}\left(\frac{n r_{1}}{p} t\right) J_{n-1}\left(\frac{n r_{2}}{p} t\right)+J_{n+1}\left(\frac{n r_{1}}{p} t\right) J_{n+1}\left(\frac{n r_{2}}{p} t\right),
$$

in which the argument, ntr/p, of the Bessel's function is large (even in the case $n=1$ ), except when $t$ is small, but in that case the factor $t^{2}$ in the integrand of eq 31 renders its values of little or no importance in the initial range of the variable $t$. (Also all the Bessel's functions involved vanish with $t$ except $J_{0}$ ). We make an infinitesimal error in $I$ by introducing in place of $J\left(n, \frac{n t}{p}\right)$ the principal term of its asymptotic expansion, which is

$$
\frac{n}{p} \sqrt{r_{1} r_{2}} J\left(n, \frac{n t}{p}\right) \approx \frac{2}{\pi t}\left[\cos \frac{n}{p}\left(r_{2}-r_{2}\right) t-(-1)^{n} \sin \frac{n}{p}\left(r_{2}+r_{1}\right) t\right] .
$$

Reference to tabular values of Bessel's functions shows that this is exceedingly accurate for arguments greater than 10. In this case, if the pitch, $2 \pi p$, is $0.1 \mathrm{~cm}$, and $r_{1}=15 \mathrm{~cm}$, the argument $n r_{1} t / p$ is $300 \pi n t$, or $t(10)^{3}$, even in the case $n=1$. Hence the asymptotic expansion is good if $t$ is as small as .01, in which case the factor $t^{2}$ of the integrand in eq 31 is not greater than $(10)^{-4}$. 
Hence, if $\lambda, r_{2}-r_{1}$, and $p$ are all infinitesimals, the integral $I$ of eq 31 may be shown by use of eq 32 to differ by an infinitesimal amount from the following:

$$
\begin{aligned}
I & =\frac{2}{\pi^{2}} \int_{0}^{\infty}\left[\frac{2 t}{\left(1+t^{2}\right)^{2}}+\frac{t}{1+t^{2}}\right] e^{-n\left|\frac{\lambda}{p}\right| t} \cos \frac{n}{p}\left(r_{2}-r_{1}\right) t d t \\
& =\frac{2}{\pi^{2}}+\frac{2}{\pi^{2}} \int_{0}^{\infty} \frac{e^{-\frac{n}{p}|\lambda| t}}{1+t^{2}}\left[\left(t-\frac{n}{p}|\lambda|\right) \cos \frac{n}{p}\left(r_{2}-r_{1}\right) t-\frac{n}{p}\left(r_{2}-r_{1}\right) \sin \frac{n}{p}\left(r_{2}-r_{1}\right) t\right] d t .
\end{aligned}
$$

This is equivalent to

$$
I=\frac{2}{\pi^{2}}+\frac{2}{\pi^{2}} \int^{\infty} \frac{s+\frac{i n z^{\prime}}{p}}{1+s^{2}} e^{\frac{i n z^{\prime}}{p} s} d s,
$$

where the real part is to be taken and $z^{\prime}$ is defined by eq $20^{\prime}$. The detailed justification of this process of deriving eq 33 is given in appendix 5 of paper 2 . In appendix 8 of the same paper, it is also shown by deforming the path of the integral that

$$
\frac{2}{\pi^{2}} \int_{0}^{\infty} \frac{s+\frac{i n z^{\prime}}{p}}{1+s^{2}} e^{\frac{i n z^{\prime}}{p} s} d s=\frac{i}{\pi}\left(1+\frac{n z^{\prime}}{p}\right) e^{\frac{-n z^{\prime}}{p}}+\frac{2}{\pi^{2}} \int_{0}^{\infty} \frac{\left[t \cos n t-n\left(\frac{z^{\prime}}{p}\right)^{2} \sin n t\right]}{t^{2}+\left(\frac{z^{\prime}}{p}\right)^{2}} d t .
$$

Hence eq 30 becomes

$$
\begin{aligned}
& -\frac{\sqrt{r_{1} r_{2}}}{\pi} \int_{0}^{\infty} \frac{1-\left(\frac{p s}{n}\right)^{2}}{\left[1+\left(\frac{p s}{n}\right)^{2}\right]^{2}} e^{-|\lambda| s} J(n, s) d s=-\frac{1}{\pi}\left[\phi_{n-1}\left(k_{\lambda}\right)+\phi_{n+1}\left(k_{\lambda}\right)\right] \\
& \quad+\frac{2}{\pi^{2}}+\frac{i}{\pi}\left(1+\frac{n z^{\prime}}{p}\right) e^{-\frac{n z^{\prime}}{p}}+\frac{2}{\pi^{2}} \int_{0}^{\infty} \frac{t \cos n t-n\left(\frac{z^{\prime}}{p}\right)^{2} \sin n t}{t^{2}+\left(\frac{z^{\prime}}{p}\right)^{2}} d t .
\end{aligned}
$$

By the same process it is found that

$$
\begin{aligned}
-\frac{2 p \sqrt{r_{1} r_{2}}}{n \pi} \int_{0}^{\infty} \frac{s e^{-|\lambda| s} J(n, s)}{\left[1+\left(\frac{p s}{n}\right)^{2}\right]^{2}} d s=-\frac{2}{\pi^{2}} \int_{0}^{\infty} \frac{1-\frac{i n z^{\prime}}{p} s}{1+s^{2}} e^{\frac{i n z z}{p}} d s \\
=-\frac{1}{\pi}\left(1+\frac{n z^{\prime}}{p}\right) e^{-\frac{n z \prime}{p}}-\frac{2 i z}{\pi^{2} p} \int_{0}^{\infty} \frac{\sin n t-n t \cos n t}{t^{2}+\left(\frac{z^{\prime}}{p}\right)^{2}} d t .
\end{aligned}
$$


Now, by eq $13 \mathrm{c}$

$$
\frac{\omega_{a}(\lambda, \alpha)}{\pi \sqrt{r_{1} r_{2}}}=\frac{1}{\pi \sqrt{r_{1} r_{2}}} \sum_{n=1}^{\infty} \omega_{n}(\lambda, \alpha),
$$

where $\omega_{n}$ is given by eq 24 and where the two integrals of eq 24 are given by eq 35 and 36 . Hence

$$
\begin{gathered}
\frac{\omega_{a}(\lambda, \alpha)}{\pi \sqrt{r_{1} r_{2}}}=\sum_{n=1}^{\infty} \frac{\cos n \alpha}{n^{2}}\left\{-\frac{1}{\pi}\left[\phi_{n-1}\left(k_{\lambda}\right)+\phi_{n+1}\left(k_{\lambda}\right)\right]+\frac{2}{\pi^{2}}+\right. \\
\left.+\frac{i}{\pi}\left(1+\frac{n z^{\prime}}{p}\right) e^{\frac{-n z^{\prime}}{p}}+\frac{2}{\pi^{2}} \int_{0}^{\infty} \frac{t \cos n t-n\left(\frac{z^{\prime}}{p}\right)^{2} \sin n t}{t^{2}+\left(\frac{z^{\prime}}{p}\right)^{2}} d t\right\} \\
\pm \sum_{n=1}^{\infty} \frac{1}{n^{2}}\left[\frac{n \lambda}{\pi p} \cos n\left(\alpha-\frac{\lambda}{p}\right)+\left(\frac{1}{\pi}+\frac{n\left|r_{2}-r_{1}\right|}{\pi p}\right) \sin n\left(\alpha-\frac{\lambda}{p}\right)\right] e^{\frac{-n\left|r_{2}-r_{1}\right|}{p}} \\
\left.-\frac{1}{\pi}\left(1+\frac{n z^{\prime}}{p}\right) e^{\frac{-n z^{\prime}}{p}} \sin n \alpha-\frac{2 i z \sin n \alpha}{\pi^{2} p} \int_{0}^{\infty} \frac{\sin n t-n t \cos n t}{t^{2}+\left(\frac{z^{\prime}}{p}\right)^{2}} d t\right\},
\end{gathered}
$$

where the real part is to be taken, $z^{\prime}$ being given by eq $20^{\prime}$. The upper or lower of the ambiguous signs belongs with $\lambda$ positive or negative, respectively.

Making some cancelations, this gives

$$
\begin{gathered}
\frac{\omega_{a}(\lambda, \alpha)}{\pi \sqrt{r_{1} r_{2}}}=\frac{2}{\pi^{2}} \sum_{n=1}^{\infty} \frac{\cos n \alpha}{n^{2}}-\frac{1}{\pi_{n=1}} \sum_{n=1}^{\infty}\left[\phi_{n-1}\left(k_{\lambda}\right)+\phi_{n+1}\left(k_{\lambda}\right)\right] \frac{\cos n \alpha}{n^{2}} \\
+\frac{1}{\pi^{2}} \int_{0}^{\infty} \frac{d t}{t^{2}+\left(\frac{z^{1}}{p}\right)^{2}}\left\{t \sum_{n=1}^{\infty} \frac{2 \cos n t \cos n \alpha}{n^{2}}-\left(\frac{z^{\prime}}{p}\right)^{2} \sum_{n=1}^{\infty} \frac{2 \sin n t \cos n \alpha}{n}\right. \\
\left. \pm \frac{i z^{\prime}}{p}\left[\sum_{n=1}^{\infty} \frac{2 \sin n t \sin n \alpha}{n^{2}}-t \sum_{n=1}^{\infty} \frac{2 \cos n t \sin n \alpha}{n}\right]\right\}
\end{gathered}
$$

It is now easy to see, by taking the real part of this equation, that the ambiguous sign \pm may be avoided, for we obtain the same real part if we replace $z^{\prime}$ by $z$ in eq 37 and take the upper of the double sign $\mp$. We thus return to the complex variable $z$ of eq 20 and have no more need for $z^{\prime}$.

It is necessary to evaluate eq 37 for the case where $-2 \pi<\alpha<2 \pi$.

Writing $2 \cos n t \cos n \alpha=\cos n(t+\alpha)+\cos n(t-\alpha)$, and then making the substitution $t^{\prime}=t+\alpha$ and $t^{\prime}=t-\alpha$, respectively, we obtain integrals 
614 Journal of Research of the National Bureau of Standards [Vol.24 over the infinite range, in which the integrand contains either the factor

$$
\pi^{-2} \sum_{1}^{\infty} n^{-2} \cos n t
$$

or

$$
\pi^{-2} \sum_{1}^{\infty} n^{-1} \sin n t
$$

both of which are periodic functions of $t$ with period $2 \pi$, the first being an even function of $t$, which is equal to

$$
\left(\frac{t}{2 \pi}\right)^{2}-\frac{t}{2 \pi}+\frac{1}{6}
$$

for the positive range, $0 \bar{\gtrless} t \bar{\gtrless} 2 \pi$; the second an odd function, which is equal to

$$
-\frac{1}{2 \pi}\left[2\left(\frac{t}{2 \pi}\right)-1\right]
$$

for this range.

Hence, writing

$$
\int_{0}^{\infty}(\ldots) d t=\sum_{n=0}^{\infty} \int_{2 \pi n}^{2 \pi(n+1)}(\ldots) d t
$$

and taking account of the periodicity of the series, we find (for the case $0 ₹|\alpha|<2 \pi)$.

$\left.I_{1} \equiv \frac{1}{\pi^{2}} \int_{0}^{\infty} \frac{d t}{t^{2}+\frac{z^{2}}{p^{2}}} \mid t \sum_{1}^{\infty} \frac{2 \cos n t \cos n \alpha}{n^{2}}-\frac{z^{2}}{p^{2}} \sum_{1}^{\infty} \frac{2 \sin n t \cos n \alpha}{n}\right\}$

$$
\begin{aligned}
& =-2 \int_{0}^{\frac{|\alpha|}{2 \pi}} \frac{\left(t-\frac{|\alpha|}{2 \pi}\right)\left(t^{2}-t+\frac{1}{6}\right)+\left(\frac{z}{2 \pi p}\right)^{2}(2 t-1)}{\left(t-\frac{|\alpha|}{2 \pi}\right)^{2}+\left(\frac{z}{2 \pi p}\right)^{2}} d t \\
& +\sum_{n=0}^{\infty} d t \frac{\left(t+n+\frac{\alpha}{2 \pi}\right)\left(t^{2}-t+\frac{1}{6}\right)+\left(\frac{z}{2 \pi p}\right)^{2}(2 t-1)}{\left(t+n+\frac{\alpha}{2 \pi}\right)^{2}+\left(\frac{z}{2 \pi p}\right)^{2}} d t
\end{aligned}
$$




$$
\left.+\frac{\left(t+n-\frac{\alpha}{2 \pi}\right)\left(t^{2}-t+\frac{1}{6}\right)+\left(\frac{z}{2 \pi p}\right)^{2}(2 t-1)}{\left(t+n-\frac{\alpha}{2 \pi}\right)^{2}+\left(\frac{z}{2 \pi p}\right)^{2}}\right\}
$$

and

$$
\begin{gathered}
\left.I_{2} \equiv \frac{-i z}{\pi^{2} p} \int_{0}^{\infty} \frac{d t}{t^{2}+\frac{z^{2}}{p^{2}}} \mid \sum_{1}^{\infty} \frac{2 \sin n t \sin n \alpha}{n^{2}}-t \sum_{1}^{\infty} \frac{\cos n t \sin n \alpha}{n}\right\} \\
= \pm 2 i\left(\frac{z}{2 \pi p}\right) \int_{0}^{\frac{|\alpha|}{2 \pi}} \frac{t^{2}-t+\frac{1}{6}-\left(t-\frac{|\alpha|}{2 \pi}\right)(2 t-1)}{\left(t-\frac{|\alpha|}{2 \pi}\right)^{2}+\left(\frac{z}{2 \pi p}\right)^{2}} d t \\
-2 i\left(\frac{z}{2 \pi p}\right) \sum_{n=0}^{\infty} \frac{d t}{t^{2}}\left\{\frac{t^{2}-t+\frac{1}{6}-\left(t+n+\frac{\alpha}{2 \pi}\right)(2 t-1)}{\left(t+n+\frac{\alpha}{2 \pi}\right)^{2}+\left(\frac{z}{2 \pi p}\right)^{2}}\right. \\
\left.-\frac{t^{2}-\frac{1}{6}-\left(t+n-\frac{\alpha}{2 \pi}\right)^{2}(2 t-1)}{\left(t+n-\frac{\alpha}{2 \pi}\right)^{2}+\left(\frac{z}{2 \pi p}\right)^{2}}\right\}
\end{gathered}
$$

where the upper sign of the $\mp$ is to be taken when $\alpha$ is positive and the lower when $\alpha$ is negative.

Hence

$$
\begin{gathered}
I_{1}+I_{2}=-2 \int_{0}^{\frac{\alpha}{2 \pi} t^{2}-|t|+\frac{1}{6}-\left(\frac{i z}{2 \pi p}\right)(2 t \mp 1)} \frac{t-\frac{p \alpha+i z}{2 \pi p}}{t} d t \\
+\sum_{n=0}^{\infty} \int_{0}^{1} d t \frac{t^{2}-t+\frac{1}{6}-\left(\frac{i z}{2 \pi p}\right)(2 t-1)}{t+n-\frac{1}{6}+\left(\frac{i z}{2 \pi p}\right)(2 t-1)} \\
+\frac{t+n+\frac{p \alpha+i z}{2 \pi p}}{2 \pi p}
\end{gathered}
$$


or

$I_{1}+I_{2}=-2 \int_{0}^{\alpha / 2 \pi}\left[t \mp 1+\frac{\alpha}{2 \pi}-\frac{i z}{2 \pi p}+\frac{\frac{1}{6} \mp \frac{\alpha}{2 \pi}+\left(\frac{\alpha}{2 \pi}\right)^{2}+\left(\frac{z}{2 \pi p}\right)^{2}}{t-\frac{p \alpha+i z}{2 \pi p}}\right] d t$

$+\sum_{n=0}^{\infty} \int_{0}^{1} d t\left(2 t-2 n-2+\frac{n(n+1)+\frac{1}{6}+(2 n+1) \frac{\alpha}{2 \pi}+\left(\frac{\alpha}{2 \pi}\right)^{2}+\left(\frac{z}{2 \pi p}\right)^{2}}{t+n+\frac{p \alpha+i z}{2 \pi p}}\right.$

$$
\left.+\frac{n(n+1)+\frac{1}{6}-(2 n+1) \frac{\alpha}{2 \pi}+\left(\frac{\alpha}{2 \pi}\right)^{2}+\left(\frac{z}{2 \pi p}\right)^{2}}{t+n-\frac{p \alpha+i z}{2 \pi p}}\right\}
$$

At this point it is convenient to introduce the complex quantity

$$
z_{12} \equiv x_{2}^{0}-x_{1}^{0}+i\left(r_{2}-r_{1}\right)=-(p \alpha+i z) .
$$

Then for brevity let

$$
t_{0} \equiv-\frac{z_{12}}{2 \pi p}=\frac{\alpha}{2 \pi}+i\left(\frac{z}{2 \pi p}\right)
$$

Then $\left|t_{0}\right|<1$, since the absolute value, $\left|z_{12}\right|$, is the distance between the two points, $P_{1}^{0}$ and $P_{2}^{0}$, in the reference sections, through which the helices $h_{1}$ and $h_{2}$ pass. The terms of the series in eq 41 may therefore be expanded before integrating. This gives

$$
\begin{gathered}
I_{1}+I_{2}=-1-\frac{\alpha}{\pi}\left[\frac{3 \alpha}{4 \pi}-\frac{i z}{2 \pi p} \mp 1\right]-2\left[\frac{1}{6} \mp \frac{\alpha}{2 \pi}+\left(\frac{\alpha}{2 \pi}\right)^{2}+\left(\frac{z}{2 \pi p}\right)^{2}\right] \int_{0}^{\alpha / 2 \pi} \frac{d t}{t-t_{0}} \\
+\left[\frac{1}{6}+\frac{\alpha}{2 \pi}+\left(\frac{\alpha}{2 \pi}\right)^{2}+\left(\frac{z}{2 \pi p}\right)^{2}\right] \int_{0}^{1} \frac{d t}{t+t_{0}} \\
+\left[\frac{1}{6}-\frac{\alpha}{2 \pi}+\left(\frac{\alpha}{2 \pi}\right)^{2}+\left(\frac{z}{2 \pi p}\right)^{2}\right] \int_{0}^{1} \frac{d t}{t-t_{0}} \\
-\sum_{n=1}^{\infty}\left\{2 n+1-\left[\frac{1}{6}+n(n+1)\right] \log \left(\frac{n+1}{n}\right)^{2}\right\} \\
+\left[\frac{1}{6}+\left(\frac{\alpha}{2 \pi}\right)^{2}+\left(\frac{z}{2 \pi p}\right)^{2}\right] \sum_{n=1}^{\infty} \sum_{s=1}^{\infty} \frac{t_{0}^{2 s}}{s}\left[\frac{1}{n^{2 s}}-\frac{1}{(n+1)^{2 s}}\right] \\
-\frac{\alpha}{\pi} \sum_{n=1}^{\infty}\left\{(2 n+1) \sum_{s=1}^{\infty} \frac{t_{0}^{2 s-1}}{2 s-1}\left[\frac{1}{n^{2 s-1}}-\frac{1}{(n+1)^{2 s-1}}\right]-t_{0} \log \left(\frac{n+1}{n}\right)^{2}\right\}
\end{gathered}
$$




$$
+\sum_{n=1}^{\infty}\left\{n(n+1) \sum_{s=1}^{\infty} \frac{t_{0}^{2 s}}{s}\left[\frac{1}{n^{2 s}}-\frac{1}{(n+1)^{2 s}}\right]-t_{0}^{2} \log \left(\frac{n+1}{n}\right)^{2}\right\}
$$

The first series in this equation is purely numerical and has the value .004 , which is negligible, as are terms 10 times as large.

The term containing the next series is found (by summing first with respect to $n$ ) to be

$$
-\left[\frac{1}{6}+\left(\frac{\alpha}{2 \pi}\right)^{2}+\left(\frac{z}{2 \pi p}\right)^{2}\right] \log \left(1-t_{0}^{2}\right) .
$$

To transform the next series in eq 44 , we expand $\log \left(\frac{n+1}{n}\right)^{2}$

and write

$\sum_{s=1}^{\infty} \frac{t^{2 s-1}}{2 s-1} B_{n}, s=(2 n+1) \sum_{s=1}^{\infty} \frac{t^{2 s-1}}{2 s-1}\left[\frac{1}{n^{2 s-1}}-\frac{1}{(n+1)^{2 s-1}}\right]-t_{0} \log \left(\frac{n+1}{n}\right)^{2}$,

where

$$
B_{n, 1} \equiv-\frac{1}{n(n+1)}+2\left[\frac{1}{2 n^{2}}-\frac{1}{3 n^{3}}+\frac{1}{4 n^{4}}-\frac{1}{5 n^{5}}+\ldots\right]
$$

and

$$
B_{n, s}=2\left[\frac{1}{n^{2 s-2}}-\frac{1}{(n+1)^{2 s-2}}\right]+\frac{1}{n^{2 s-1}}+\frac{1}{(n+1)^{2 s-1}}, \text { if } s>1 .
$$

Hence

$$
\begin{aligned}
\sum_{n=1}^{\infty} B_{n}, s & =1+2 \sum_{n=1}^{\infty} \frac{1}{n^{2 s-1}}, \text { if } s>1 . \\
& =.154 \ldots \ldots, \text { if } s=1 .
\end{aligned}
$$

Hence introducing Riemann's zeta function

$$
\zeta(s) \equiv \sum_{n=1}^{\infty} \frac{1}{n^{s}} \text { for } s>1,
$$

then the next to last series in eq 44 is

$$
\frac{\alpha}{\pi}\left\{.846 t_{0}+\frac{1}{2} \log \left(\frac{1-t_{0}}{1+t_{0}}\right)-2 \sum_{s=2}^{\infty} \frac{\zeta(2 s-1)}{2 s-1} \cdot t_{0}^{2 s-1}\right\} .
$$

Similarly, to transform the last series in eq 44, we write

$$
\sum_{s=1}^{\infty} \frac{t_{0}^{2 s}}{s} C_{n}, s=n(n+1) \sum_{s=1}^{\infty} \frac{t_{0}^{2 s}}{s}\left[\frac{1}{n^{2 s}}-\frac{1}{(n+1)^{2 s}}\right]-t_{0}^{2} \log \left(\frac{n+1}{n}\right)^{2},
$$


where $C_{n, 1}=B_{n, 1}$ and

Hence

$$
C_{n, s}=\frac{1}{n^{2 s-2}}-\frac{1}{(n+1)^{2 s-2}}+\frac{1}{n^{2 s-1}}+\frac{1}{(n+1)^{2 s-1}}, \text { if } s>1 .
$$

$$
\begin{aligned}
\sum_{n=1}^{\infty} C_{n}, & =2 \sum_{n=1}^{\infty} \frac{1}{n^{2 s-1}}=2 \zeta(2 s-1), \text { if } s>1 \\
& =.154, \text { if } s=1 .
\end{aligned}
$$

The last series in eq 44 is therefore

Hence eq 44 becomes

$$
.154 t_{0}^{2}+2 \sum_{s=2}^{\infty} \frac{\zeta(2 s-1)}{s} t_{0}^{2 s}
$$

$$
\begin{aligned}
I_{1}+I_{2}=- & +\frac{|\alpha|}{\pi}\left(1-\frac{|\alpha|}{2 \pi}\right)-\frac{\alpha}{2 \pi}\left[\frac{\alpha}{2 \pi}-\frac{2 i z}{2 \pi p}\right] \\
& -2\left[\frac{1}{6}-\frac{|\alpha|}{2 \pi}+\left(\frac{\alpha}{2 \pi}\right)^{2}+\left(\frac{z}{2 \pi p}\right)^{2}\right] \cdot\left[\log \left(\frac{z}{2 \pi p t_{0}}\right)+i \frac{\pi}{2}\right] \\
& +\left[\frac{1}{6}+\frac{\alpha}{2 \pi}+\left(\frac{\alpha}{2 \pi}\right)^{2}+\left(\frac{z}{2 \pi p}\right)^{2}\right] \cdot\left[\log \left(1+t_{0}\right)-\log t_{0}\right] \\
& +\left[\frac{1}{6}-\frac{\alpha}{2 \pi}+\left(\frac{\alpha}{2 \pi}\right)^{2}+\left(\frac{z}{2 \pi p}\right)^{2}\right] \cdot\left[\log \left(1-t_{0}\right)-\log t_{0}+i \pi\right] \\
& -\left[\frac{1}{6}+\left(\frac{\alpha}{2 \pi}\right)^{2}+\left(\frac{z}{2 \pi p}\right)^{2}\right] \log \left(1-t_{0}^{2}\right)+\frac{\alpha}{2 \pi} \log \frac{1-t_{0}}{1+t_{0}} \\
+ & {[1-154] \frac{\alpha t_{0}}{\pi}+.154 t_{0}^{2}-\frac{2 \alpha}{\pi} \sum_{s=2}^{\infty} \frac{\zeta(2 s-1}{2 s-1} \cdot t_{0}^{2 s-1}+2 \sum_{s=2}^{\infty} \frac{\zeta(2 s-1)}{s} \cdot t_{0}^{2 s}, }
\end{aligned}
$$

where imaginary terms are to be discarded.

This reduces to

$$
\begin{aligned}
I_{1}+I_{2}=-1 & -\frac{3 \alpha^{2}}{4 \pi^{2}}+\frac{|\alpha|}{\pi}\left(1-\frac{|\alpha|}{\pi}\right)+1.85 \frac{\alpha}{\pi} t_{0}+.154 t_{0}^{2} \\
& +\left[\frac{|\alpha|}{\pi}\left(1-\frac{|\alpha|}{2 \pi}\right)-\frac{1}{3}-2\left(\frac{z}{2 \pi p}\right)^{2}\right] \log \frac{z}{2 \pi p} \\
& -\frac{|\alpha|}{\pi} \log t_{0}-2 \frac{\alpha}{\pi} \sum_{s=2}^{\infty} \frac{\zeta(2 s-1)}{2 s-1} t_{0}^{2 s-1}+2 \sum_{s=2}^{\infty} \frac{\zeta(2 s-1)}{s} t_{0}^{2 s} .
\end{aligned}
$$

The definitions of $I_{1}$ and $I_{2}$ in eq 38 and 39 show that eq 37 is $\frac{\omega_{a}(\lambda, \alpha)}{\pi \sqrt{r_{1} r_{2}}}=\frac{2}{\pi^{2}} \sum_{n=1}^{\infty} \frac{\cos n \alpha}{n^{2}}-\frac{1}{\pi} \sum_{n=1}^{\infty}\left[\phi_{n-1}\left(k_{\lambda}\right)+\phi_{n+1}\left(k_{\lambda}\right)\right] \frac{\cos n \alpha}{n^{2}}+I_{1}+I_{2}$ 
Since we are considering the case where $-2 \pi<\alpha<2 \pi$ for which eq 46 applies, it is evident by reference to eq 28 and $28^{\prime \prime}$ that

$$
\frac{2}{\pi^{2}} \sum_{n=1}^{\infty} \frac{\cos n \alpha}{n^{2}}=\frac{1}{3}-\frac{|\alpha|}{\pi}\left(1-\frac{|\alpha|}{2 \pi}\right) .
$$

Also since $\lambda$ is small, as well as $r_{2}-r_{1}$, it is evident that $k^{\prime}{ }_{\lambda}$ is small, so that by eq 27 , neglecting infinitesimals

$$
\begin{gathered}
-\frac{1}{\pi} \sum_{n=1}^{\infty}\left[\phi_{n-1}\left(k_{\lambda}\right)+\phi_{n+1}\left(k_{\lambda}\right)\right] \frac{\cos n \alpha}{n^{2}}=-\frac{\pi}{3} \phi_{1}\left(k_{\lambda}\right)-\frac{1}{2}\left|\sin \frac{\alpha}{2}\right| \cdot\left(1-\log \frac{2\left|\sin \frac{\alpha}{2}\right|}{k_{\lambda}^{\prime}}\right) \\
=\frac{2}{3}+\frac{1}{3} \log \frac{k_{\lambda}^{\prime}}{4}-\frac{1}{2}\left|\sin \frac{\alpha}{2}\right| \cdot\left(1-\log \frac{2\left|\sin \frac{\alpha}{2}\right|}{k_{\lambda}^{\prime}}\right) \\
=\frac{2}{3}-\frac{1}{2} \mid \sin \frac{\alpha}{2}\left(1-\log \frac{1}{2}\left|\sin \frac{\alpha}{2}\right|\right)+\left[\frac{1}{3}-\frac{1}{2}\left|\sin \frac{\alpha}{2}\right|\right] \log \left(\frac{z}{8 \sqrt{r_{1} r_{2}}}\right) .
\end{gathered}
$$

By eq $28^{\prime}$ and $28^{\prime \prime}$ we may place

so that

$$
\frac{1}{2}\left|\sin \frac{\alpha}{2}\right|=\frac{|\alpha|}{\pi}\left(1-\frac{|\alpha|}{2 \pi}\right)
$$

$$
\begin{aligned}
-\frac{1}{\pi} \sum_{n=1}^{\infty}\left[\phi_{m-1}\left(k_{\lambda}\right)+\right. & \left.\phi_{n+1}\left(k_{\lambda}\right)\right] \frac{\cos n \alpha}{n^{2}}=\frac{2}{3}-\frac{|\alpha|}{\pi}\left(1-\frac{|\alpha|}{2 \pi}\right)\left[1-\log \frac{|\alpha|}{\pi}\left(1-\frac{|\alpha|}{2 \pi}\right)\right] \\
+ & {\left[\frac{1}{3}-\frac{|\alpha|}{\pi}\left(1-\frac{|\alpha|}{2 \pi}\right)\right] \log \frac{z}{8 \sqrt{r_{1} r_{2}}} \cdot }
\end{aligned}
$$

Using eq 46,48 , and 49 , the eq 47 becomes

$$
\begin{gathered}
\frac{\omega_{\alpha}(\lambda, \alpha)}{\pi \sqrt{r_{1} r_{2}}}=1.85 \frac{\alpha}{\pi} t_{0}+.15 t_{0}^{2}-\frac{|\alpha|}{\pi} \log t_{0} \\
+2 \sum_{s=2}^{\infty} \frac{\zeta(2 s-1)}{s} t_{0}^{2}-2 \frac{\alpha}{\pi} \sum_{s=2}^{\infty} \frac{\zeta(2 s-1)}{2 s-1} t_{0}^{2 s-1} \\
-\frac{3 \alpha^{2}}{4 \pi^{2}}-2\left(\frac{z}{2 \pi p}\right)^{2} \log \frac{z}{2 \pi p} \\
-\frac{|\alpha|}{\pi}\left(1-\frac{|\alpha|}{2 \pi}\right)\left[1-\log \frac{|\alpha|}{\pi}\left(1-\frac{|\alpha|}{2 \pi}\right) \frac{4 \sqrt{r_{1} r_{2}}}{\pi p}\right]-\frac{1}{3} \log \frac{4 \sqrt{r_{1} r_{2}}}{\pi p}
\end{gathered}
$$

On replacing $t_{0}$ by its value $-\frac{z_{12}}{2 \pi p}$ and $\sqrt{r_{1} r_{2}}$ by $a$, this gives 


$$
\begin{aligned}
& -\frac{2 \omega_{a}(\lambda, \alpha)}{\pi a}=3.7\left(\frac{\alpha}{\pi}\right)\left(\frac{z_{12}}{2 \pi p}\right)-\cdot 3\left(\frac{z_{12}}{2 \pi p}\right)^{2}+2 \frac{|\alpha|}{\pi} \log \frac{z_{12}}{2 \pi p} \\
& -4 \sum_{s=2}^{\infty} \frac{\zeta(2 s-1)}{s}\left(\frac{z_{12}}{2 \pi p}\right)^{2 s}-4 \frac{\alpha}{\pi} \sum_{s=2}^{\infty} \frac{\zeta(2 s-1)}{2 s-1}\left(\frac{z_{12}}{2 \pi p}\right)^{2 s-1}+4\left(\frac{z}{2 \pi p}\right)^{2} \log \left(\frac{z}{2 \pi p}\right) \\
& +\frac{3 \alpha^{2}}{2 \pi^{2}}+\frac{2|\alpha|}{\pi}\left(1-\frac{|\alpha|}{2 \pi}\right)\left\{1-\log \left[\frac{|\alpha|}{\pi}\left(1-\frac{|\alpha|}{2 \pi}\right)\left(\frac{8 a}{2 \pi p}\right)\right]\right\}+\frac{2}{3} \log \frac{8 a}{2 \pi p} \cdot
\end{aligned}
$$

The imaginary part is to be discarded. This equation is valid for $\left|z_{12}\right|<2 \pi p$ and for $-2 \pi<\alpha<2 \pi$. It shows that $\omega_{a}(\lambda, \alpha)$ contributes a correction term which is of the smallest order of magnitude retained.

To express the first logarithmic term in eq 29 in terms of the variable $z_{12}$ defined in eq 42 , we first let $v=\left|r_{2}-r_{1}\right|+i\left(x_{2}^{0}-x_{1}^{0}\right)$, so that, as in appendix 7 of paper 2

$$
-\log \left(1-e^{-\frac{v}{p}}\right)=\frac{v}{2 p}-\log \frac{v}{p}+\sum_{s=1}^{\infty} \frac{\zeta(2 s)}{s}\left(\frac{i v}{2 \pi p}\right)^{2 s} \text {, if }|v|<2 \pi p .
$$

Since the real part of this is to be taken (this logarithm contains a real factor in eq 29), we may place

$$
\begin{gathered}
\log |v|=\log \left|z_{12}\right| \text { and } \mathscr{R}(i v)^{2 s}=\mathscr{R}\left(z_{12}^{2 s},\right) \\
\text { since } \mathscr{R}(i v)^{2 s}=\mathscr{R}\left[-\left(x_{2}^{0}-x_{1}^{0}\right)+i\left|r_{2}-r_{1}\right|\right]^{2 s},
\end{gathered}
$$

and this contains only even powers of $-\left(x_{2}^{0}-x_{1}^{0}\right)$ and $\left|r_{2}-r_{1}\right|$. The eq 29 therefore becomes

$$
\begin{aligned}
& \omega_{a}(l+\lambda, \alpha)+\omega_{a}(-l+\lambda, \alpha)=\frac{l\left|r_{2}-r_{1}\right| \sqrt{r_{1} r_{2}}}{p^{2}} \\
& -4 \pi N a\left[\log \frac{z_{12}}{p}-\sum_{s=1}^{\infty} \frac{\zeta(2 s)}{s}\left(\frac{z_{12}}{2 \pi p}\right)^{2 s}\right] \cdot\left[1+\frac{r_{1}-a+r_{2}-a}{2 a}\right] \\
& -\pi a\left\{\frac{2 \pi}{3} \phi_{1}(k)+\frac{1}{k} \sqrt{1-k^{2} \cos ^{2} \frac{\alpha}{2}}\right. \\
& \left.-\left(\frac{k^{\prime}}{k}\right)^{2}\left(\cos \frac{\alpha}{2}\right) \sin ^{-1}\left(k \cos \frac{\alpha}{2}\right)-\frac{1}{k^{2}} \sin \frac{\alpha}{2} \log \left[\frac{k \sin \frac{\alpha}{2}+\sqrt{1-k^{2} \cos ^{2} \frac{\alpha}{2}}}{k^{\prime}}\right]\right\},
\end{aligned}
$$

where $k_{l}$ has been replaced by $k$ and $\sqrt{r_{1} r_{2}}$ by $a$ in those terms which are of second order. In the term multiplied by $4 \pi N$, we have replaced $\sqrt{r_{1} r_{2}}$ by $a\left[1+\frac{r_{1}-a+r_{2}-a}{2 a}\right]$. 


\section{SECOND-ORDER APPROXIMATION FOR $\mathrm{m}$}

By use of eq 18,21,50, and 51 in the eq 15 , we obtain the secondorder approximation to $m$ as the real part of eq 53 below. In this equation, we have placed

$$
\mathrm{z}_{12}=\mathrm{z}_{2}-\mathrm{z}_{1}, \text { where }\left\{\begin{array}{l}
\mathrm{z}_{1}=x_{1}^{0}+i\left(r_{1}-a_{1}\right) \\
\mathrm{z}_{2}=x_{2}^{0}+i\left(r_{2}-a_{1}\right)
\end{array}\right\} .
$$

The complex quantities, $z_{1}$ and $z_{2}$, will be found to be the most appropriate ones with respect to integration over the sections of the wires. They are both referred to the centroid, $0_{1}$, of section $S_{1}$ as origin, but could equally well have been defined with reference to any other point, such as $0_{2}$. As shown by figure 1 , the position of any point $P_{1}\left(x_{1}^{0}, r_{1}\right)$ in section $S_{1}$ is represented by the vector or complex variable $z_{1}$, and the position of any point $P_{2}\left(x_{2}^{0}, r_{2}\right)$ in section $S_{2}$ by the vector or complex variable $z_{2}$ with reference to the same origin. Since $P_{1}$ and $P_{2}$ are in different wires, it is evident that $\left|z_{2}-z_{1}\right| / 2 \pi p$ is less than 1.

The equation for $m$ takes the form

$$
\begin{gathered}
m_{\alpha}\left(x_{1}^{0}, r_{1} ; x_{2}^{0}, r_{2}\right)=L_{s}+2 \omega_{x}(l) \\
+2 \pi a\left\{C_{\alpha}+C_{1}\left(\frac{x_{2}^{0}-x_{1}^{0}}{2 \pi p}\right)+C_{2}\left(\frac{x_{2}^{0}-x_{1}^{0}}{2 \pi p}\right)^{2}+C_{3}\left(\frac{r_{2}-r_{1}}{2 \pi p}\right)^{2}+\frac{|\alpha|}{\pi} \log \left(\frac{z_{2}-z_{1}}{2 \pi p}\right)\right. \\
+16 A_{1}\left(\frac{a}{2 \pi p}\right)\left(\frac{r_{1}-a_{1}+r_{2}-a_{2}}{2 \pi p}\right)+16 A_{3}\left(\frac{r_{1}-a_{1}}{2 \pi p}\right)\left(\frac{r_{2}-a_{2}}{2 \pi p}\right) \\
\left.-2 N\left(1+\frac{r_{1}-a_{1}+r_{2}-a_{2}}{2 \pi p}\right) f\left(\frac{z_{2}-z_{1}}{2 \pi p}\right)+f_{1}\left(\frac{z_{2}-z_{1}}{2 \pi p}\right)+\frac{\alpha}{\pi} f_{2}\left(\frac{z_{2}-z_{1}}{2 \pi p}\right)\right\},
\end{gathered}
$$

where

$$
\begin{aligned}
& C_{1}=\frac{\alpha}{\pi}\left[2.85+2 \pi \phi_{1}-2 \log \frac{4 a}{\pi p}\right] \\
& C_{2}=.85+2 \pi \phi_{1}-2 \log \frac{4 a}{\pi p} \\
& C_{3}=.15+16 A_{4}+2 \log \frac{4 a}{\pi p} .
\end{aligned}
$$

The constants $A_{1}, A_{3}, A_{4}$, and $\phi_{1}$ are functions of $k$, which are defined in eq 19 and 8 , respectively.

$$
\begin{aligned}
& C_{\alpha}=\frac{|\alpha|}{\pi}\left(1-\frac{|\alpha|}{2 \pi}\right)\left[1-\log \frac{|\alpha|}{\pi}\left(1-\frac{|\alpha|}{2 \pi}\right)\right]+\frac{\alpha^{2}}{\pi^{2}}\left(1+\frac{\pi \phi_{1}}{2}\right) \\
& -\frac{\pi}{3} \phi_{1}-\frac{1}{2 k} \sqrt{1-k^{2} \cos ^{2} \frac{\alpha}{2}}+\frac{1}{2}\left(\frac{k^{\prime}}{k}\right)^{2}\left(\cos \frac{\alpha}{2}\right) \sin ^{-1}\left(k \cos \frac{\alpha}{2}\right) \\
& +\left(\frac{1}{3}-\frac{|\alpha|}{\pi}\right) \log \left(\frac{4 a}{\pi p}\right)+\frac{\sin \frac{\alpha}{2}}{2 k^{2}} \log \left[\frac{k \sin \frac{\alpha}{2}+\sqrt{1-k^{2} \cos ^{2} \frac{\alpha}{2}}}{k^{\prime}}\right] \text {, }
\end{aligned}
$$

where $-\pi<\alpha<\pi$. 
The real part of the complex terms in eq 53 is to be taken, so that the principal branch of the multiple-valued function $\log \left(z_{2}-z_{1}\right)$ may be understood, since this is indeterminate only to the extent of an additive constant which is a multiple of $2 \pi i$. With this understanding, this term and all the three other functions of $\left(z_{2}-z_{1}\right) / 2 \pi p$ are single-valued, analytic functions of either variable $z_{1}$ or $z_{2}$ in a region including the respective sections $S_{1}$ or $S_{2}$. To define the other functions with general argument $z$ (where $z$ is later to be replaced by $\left.\left(z_{2}-z_{1}\right) / 2 \pi p\right)$, we have the following series:

$$
f(z) \equiv \log 2 \pi z-\sum_{s=1}^{\infty} \frac{\zeta(2 s)}{s} z^{2 s}, \text { when }|z|<1,
$$

where $\log 2 \pi z$ denotes the principal branch (which is real when $z$ is positive).

$$
\begin{aligned}
& f_{1}(z) \equiv-2 \sum_{s=2}^{\infty} \frac{\zeta(2 s-1)}{s} z^{2 s}, \text { when }|z|<1 \\
& f_{2}(z) \equiv-2 \sum_{s=2}^{\infty} \frac{\zeta(2 s-1)}{2 s-1} z^{2 s-1}, \text { when }|z|<1 .
\end{aligned}
$$

Obviously,

$$
f_{1}^{\prime}(z)=2 z f_{2}^{\prime}(z) \text {, }
$$

so that

$$
f_{1}(z)=2 \int_{0}^{z} z^{\prime} f_{2}\left(z^{\prime}\right) d z^{\prime}=2 z f_{2}(z)-2 \int_{0}^{z} f_{2}\left(z^{\prime}\right) d z^{\prime} .
$$

For integrating over sections not closely resembling circles, it will in general be more convenient to use these functions in the form of series. For circular sections, the use of the series for $f$ and $f_{2}$ may be avoided, as these are summable in terms of known functions as follows:

Taking the logarithm of both sides of the equation

gives

$$
\frac{\sin \pi z}{\pi z}=\prod_{n=1}^{\infty}\left(1-\frac{z^{2}}{n^{2}}\right)
$$

$$
\log \frac{\sin \pi z}{\pi z}=\sum_{n=1}^{\infty} \log \left(1-\frac{z^{2}}{n^{2}}\right)
$$

Since we are here concerned with the case $|z|<1$, we may place

so that

$$
\log \left(1-\frac{z^{2}}{n^{2}}\right)=-\sum_{s=1}^{\infty} \frac{z^{2 s}}{s} \cdot \frac{1}{n^{2 s}}
$$

$$
\log \frac{\sin \pi z}{\pi z}=-\sum_{n=1}^{\infty} \sum_{s=1}^{\infty} \frac{z^{2 s}}{s} \frac{1}{n^{2 s}}=-\sum_{s=1}^{\infty} \frac{z^{2 s}}{s} \sum_{n=1}^{\infty} \frac{1}{n^{2 s}},
$$


the inversion of the order of summation of the double series being valid because it is absolutely convergent. Since the zeta-function of Riemann is defined by

this becomes

$$
\zeta(s)=\sum_{n=1}^{\infty} \frac{1}{n^{s}}, \text { (when) } s>1,
$$

$$
\log \frac{\sin \pi z}{\pi z}=-\sum_{s=1}^{\infty} \frac{\zeta(2 s)}{s} z^{2 s}, \text { when }|z|<1 .
$$

Comparing this with eq 53e shows that if the logarithm denotes that branch which is real when its subject is positive,

Similarly, we find that

$$
f(z)=\log 2 \sin \pi z .
$$

$$
f_{2}(z)=2 \gamma z+\log \frac{\Gamma(1+z)}{\Gamma(1-z)}
$$

where $\gamma=0.577216=$ Euler's constant.

This may be found by taking the logarithm of the infinite product which represents the reciprocal of the gamma-function

$$
\frac{1}{\Gamma(z+1)}=e^{\gamma z} \prod_{n=1}^{\infty}\left(1+\frac{z}{n}\right) e^{-\frac{z}{n}}
$$

This gives

$$
\gamma z+\log \Gamma(z+1)=-\sum_{n=1}^{\infty}\left[\log \left(1+\frac{z}{n}\right)-\frac{z}{n}\right]
$$

When

$$
|z|<1,-\left[\log \left(1+\frac{z}{n}\right)-\frac{z}{n}\right]=\sum_{s=2}^{\infty} \frac{(-1)^{s} z^{s}}{s n^{s}}
$$

so that

$$
\begin{aligned}
\gamma z+\log \Gamma(z+1) & =\sum_{s=2}^{\infty} \frac{(-1)^{s} \zeta(s)}{s} z^{s} \\
& =\sum_{s=1}^{\infty} \frac{\zeta(2 s)}{2 s} z^{2 s}-\sum_{s=2}^{\infty} \frac{\zeta(2 s-1)}{2 s-1} z^{2 s-1} \\
& =-\frac{1}{2} \log \frac{\sin \pi z}{\pi z}+\frac{1}{2} f_{2}(z) \\
& =+\frac{1}{2} \log \Gamma(1+z)+\frac{1}{2} \log \Gamma(1-z)+\frac{1}{2} f_{2}(z) s
\end{aligned}
$$

since $\Gamma(1+z) \Gamma(1-z)=\pi z / \sin \pi z$. This proves eq $53 \mathrm{k}$. 
The principal term in eq 53 is the term $L_{s}$, representing the self inductance of a cylindrical current sheet, for as shown by eq 16 , it contains the large factor $N^{2}$ or $l^{2} /(2 \pi p)^{2}$. The correction terms of first order are two terms within the braces, the first being $-2 \mathrm{Nf}$ on account of the large factor $N$. The second is the term containing $A_{1}$ with the large factor $a / 2 \pi p$. All other terms are of second order. The modulus, $k$, (complementary modulus $k^{\prime}$ ) is computed in terms of $l=2 \pi p N$ and $D=2 a$, by $k^{2}=D^{2} /\left(l^{2}+D^{2}\right)$. The cylindrical radius $a$ will be taken as the mean of the two mean cylindrical radii of the wires in the sense that $a_{1}$ and $a_{2}$ are the distances of the centroids of the reference sections from the axis.

However, it is important to notice that eq 53 represents the mutual inductance, $m_{\alpha}\left(x_{1}^{0} r_{1} ; x_{2}^{0}, r_{2}\right)$, of any two helices, $h_{1}$ and $h_{2}$, with relative azimuth $\alpha$, when the pair of coordinates $\left(x_{1}^{0}, r_{1}\right)$ and $\left(x_{2}^{0}, r_{2}\right)$, which serve to specify $h_{1}$ and $h_{2}$ are such that $\left|x_{2}^{0}-x_{1}^{0}\right|<2 \pi p,\left|\alpha_{1}\right|<\pi$ and $\left|r_{2}-r_{1}\right|$ is of the order of magnitude of $2 \pi p$. More precisely $\left|z_{2}-z_{1}\right|<2 \pi p$. It, of course, contains no reference to wire sections. Hence the constant $a$ is, within certain small limits, still at our disposal. The constants $a_{1}$ and $a_{2}$ occur only in the first-order terms of eq 53, and there they appear in the sum, $a_{1}+a_{2}$, which is $2 a$.

The only thing that has been assumed concerning $a$ in obtaining the approximations in eq 53 is that $r_{1}-a$ and $r_{2}-a$ are of the order of magnitude of the pitch. It is conceivable that for example the mean radius $a_{1}$ of the first wire section might be measurable with greater precision than $a_{2}$. It might then suit our purpose to take $a=a_{1}$, which would then be used in determining the modulus $k$ upon which all the constants $L_{s}, 2 w_{x}(l), C_{\alpha}, C_{1}$, etc. depend.

On placing $\alpha=0$ in eq 53, it is found to be equivalent to the equation which was used in paper 3 for the self inductance of a helical wire (neglecting $D / 4$, which, as indicated above, is in general less than 1 part in a million).

\section{MUTUAL INDUCTANCE, $M_{\alpha}$, OF THE HELICAL WIRES}

In evaluating the integral of eq 4 , which gives $M_{\alpha}$, there is an enormous saving of labor in adhering to the compex form until the integrations have been performed. Accordingly, we introduce the complex constant, $z_{0}$, which represents the vector from the centroid $0_{1}$ of the reference section $S_{1}$ to the centroid $0_{2}$ of reference section $S_{2}$. Its axial component is seen to be $x_{0_{2}}^{0}$ of figure 1 , which may now be designated by $x_{0}$. Its radial component is $a_{2}-a_{1}$.

Hence

$$
z_{0}=x_{0}+i\left(a_{2}-a_{1}\right)=q e^{i \beta}, \text { where }-\pi<\beta<\pi,
$$

so that $g=\left|z_{0}\right|=\sqrt{x_{0}^{2}+\left(a_{2}-a_{1}\right)^{2}}$, and $\beta=\tan ^{-1}\left[\left(a_{2}-a_{1}\right) / x_{0}\right]$. The points of integration, $P_{1}$ and $P_{2}$, are fixed respectively by the vectors $z_{1}$ and $z_{2}$, both drawn from $0_{1}$ as origin. The eq 53 then give

$$
\begin{aligned}
& z_{1}=x_{1}^{0}+i\left(r_{1}-a_{1}\right)=\rho_{1} e^{i \phi_{1}} \\
& \left.z_{2}=x_{2}^{0}+i\left(r_{2}-a_{1}\right)=z_{0}+\rho_{2} e^{i \phi_{2}}\right\}
\end{aligned}
$$


Since each wire carries unit current, as implied by eq 3 , it is evident that the constant terms in eq 53 , namely, $L_{s}+2 w_{x}(l)+2 \pi a C_{\alpha}$, when used in the integral 4 are merely multiplied by unity, whatever the shape of the sections $S_{1}$ and $S_{2}$.

All of the terms of eq 53, which are of second order, are then to be averaged first over one section, and this result then averaged over the other section. Since the points $0_{1}$ and $0_{2}$ are centroids of the reference sections, it is evident that the average value of $x_{1}^{0}$ over $S_{1}$ is zero, and the average value of $x_{2}^{0}$ over $S_{2}$ is $x_{0}$. The average of $\left(x_{1}^{0}\right)^{2}$ over $S_{1}$ may be denoted by $k_{x x}$, and that of $\left(x_{2}^{0}-x_{0}\right)^{2}$ over $S_{2}$ by $k^{\prime}{ }_{x x}$. Also the average of $r_{1}-a_{1}$ over $S_{1}$ is zero, as is the average of $r_{2}-a_{2}$ over $S_{2}$. We may denote by $k_{y v}$ the average of $\left(r_{1}-a_{1}\right)^{2}$ over $S_{1}$, and by $k_{y y}^{\prime}$ the average of $\left(r_{2}-a_{2}\right)^{2}$ over $S_{2}$. These averages multiplied by the area of the section represent its moment of inertia. For the products of inertia, we use the similar notation, where $k_{x \nu}$ denotes the average over $S_{1}$ of $x_{1}^{0}\left(r_{1}-a_{1}\right)$ and $k^{\prime}{ }_{x y}$ the average over $S_{2}$ of $\left(x_{2}^{0}-x_{0}\right)$ $\left(r_{2}-a_{2}\right)$.

Then $k_{x x}, k_{y y}, k_{x y}$, etc. are all of the order of magnitude of $(2 \pi p)^{2}$ or $x_{0}^{2}$.

The terms of first order in eq 53 must be treated more carefully. They are the two terms in 53 affected by the factors $A_{1} a / 2 \pi p$ and $-2 N$, respectively. For these we use the expressions $5 \mathrm{a}$, and for the formulation in the case of general shapes of section we prefer to use rectangular rather than polar coordinates in harmony with the definitions adopted for $k_{x x}$, etc.

The formula for the mutual inductance, $M_{\alpha}$, of two helical wires with general shape of reference section may then be written.

$$
\begin{aligned}
& M_{\alpha}=L_{s}+2 \omega_{x}(l) \\
& +2 \pi a\left\{C_{\alpha}+C_{1}\left(\frac{x_{0}}{2 \pi p}\right)+C_{2} \frac{x_{0}^{2}+k_{x x}+k_{x x}^{\prime}}{(2 \pi p)^{2}}+C_{3} \frac{k_{y y}+k_{y y}^{\prime}}{(2 \pi p)^{2}}\right. \\
& +16 A_{1} a\left[\frac{c_{x_{1}} k_{x y}+c_{x_{2}} k_{x_{y}}^{\prime}+c_{r_{1}} k_{y y}+c_{r_{2}} k_{y y}^{\prime}}{(2 \pi p)^{2}}\right] \\
& -2 N \overline{\bar{f}}+\overline{\overline{f_{1}}}+\frac{\alpha}{\pi} \overline{\overline{f_{2}}}+\frac{|\alpha|}{\pi} \overline{\overline{\log }}\left(\frac{z_{2}-z_{1}}{2 \pi p}\right) \\
& -2 N \iint \frac{d S_{1}}{S_{1}} \iint \frac{d S_{2}}{S_{2}} f\left(\frac{z_{2}-z_{1}}{2 \pi p}\right)\left[c_{x_{1}} x_{1}^{0}+c_{x_{2}}\left(x_{2}^{0}-x_{0}\right)\right. \\
& \left.+\left(c_{r_{1}}+\frac{1}{2 \pi p}\right)\left(r_{1}-a_{1}\right)+\left(c_{r 2}+\frac{1}{2 \pi p}\right)\left(r_{2}-a_{2}\right)\right] \text {, }
\end{aligned}
$$

where $\overline{\bar{f}}$, etc. denotes the double average taken in succession over the one section and then over the other, of the function of $\left(z_{2}-z_{1}\right) / 2 \pi p$.

The principal correction term comes from $-2 N \overline{\bar{f}}$, since this is the only term of first order in eq 55.

In the case of circular reference sections $k_{x y}=0$ and $k_{x x}=k_{y y}=d_{1}^{2} / 16$ so this becomes

$$
\begin{gathered}
M_{\alpha}=L_{s}+2 \omega_{x}(l)+2 \pi a\left\{C_{\alpha}+\frac{2 \alpha}{\pi}\left(\frac{x_{0}}{2 \pi p}\right)\right. \\
+\left(\frac{x_{0}}{2 \pi p}\right)\left(\frac{x_{0}+2 p \alpha}{2 \pi p}\right)\left[.85+2 \pi \phi_{1}-2 \log \left(\frac{4 a}{\pi p}\right)\right]+
\end{gathered}
$$




$$
\begin{gathered}
+\frac{1}{4}\left(\frac{E}{k}-1\right)\left[\left(1+2 a c_{r 1}\right)\left(\frac{d_{1}}{2 \pi p}\right)^{2}+\left(1+2 a c_{r 2}\right)\left(\frac{d_{2}}{2 \pi p}\right)^{2}\right] \\
-2 N \bar{f}+\overline{f_{1}}+\frac{\alpha}{\pi} f_{2}+\frac{|\alpha|}{\pi} \overline{\log }\left(\frac{z_{2}-z_{1}}{2 \pi p}\right) \\
-2 N \iint \frac{d S_{1}}{S_{1}} \iint \frac{d S_{2}}{S_{2}} f\left(\frac{z_{2}-z_{1}}{2 \pi p}\right)\left[c_{x_{1}} \rho_{1} \cos \phi_{1}+\left(c_{r 1}+\frac{1}{2 \pi p}\right) \rho_{1} \sin \phi_{1}\right. \\
\left.\left.+c_{x_{2} \rho_{2}} \cos \phi_{2}+\left(c_{r 2}+\frac{1}{2 \pi p}\right) \rho_{2} \sin \phi_{2}\right]\right\}, \quad \text { (56) } \\
\text { where } d S_{1}=\rho_{1} d \rho_{1} d \phi_{1} \text { and } S_{1}=\frac{\pi d_{1}^{2}}{4}
\end{gathered}
$$

To perform the remaining integrations, consider the line average taken around any circular arc $\rho_{1}=$ const., of any monogenic function of $z_{1}$, multiplied by $e^{i n \phi_{1}}$. Considering $n$ an integer, positive, negative, or zero,

$$
\begin{gathered}
\frac{1}{2 \pi} \int_{-\pi}^{\pi} e^{i n \phi_{1}} F\left(z_{1}\right) d \phi_{1}=\frac{1}{2 \pi i} \int_{-\pi}^{\pi} \frac{e^{i n \phi_{1}} F\left(z_{1}\right)}{\rho_{1} e^{1 \phi_{1}}} d\left(\rho_{1} e^{1 \phi_{1}}\right) \\
\quad=\frac{\rho_{1}{ }^{-n}}{2 \pi i} \oint_{1} z_{1}^{n-1} F\left(z_{1}\right) d z_{1}, \text { since } z_{1}=\rho_{1} e^{i \phi_{1}}
\end{gathered}
$$

This is a contour integral around a circle in the $z_{1}$-plane, which encloses the point $z_{1}=0$. Hence by Cauchy's theorem

$$
\begin{aligned}
\frac{1}{2 \pi} \int_{-\pi}^{\pi} e^{i n \phi_{1}} F\left(z_{1}\right) d \phi_{1} & =0, \text { if } n \text { is a positive integer } \\
& =F(0), \text { if } n=0 .
\end{aligned}
$$

and

where

$$
\frac{1}{2 \pi} \int_{-\pi}^{\pi} e^{-i n \phi_{1}} F\left(z_{1}\right) d \phi_{1}=\frac{\rho_{1}^{n} F^{(n)}(0)}{n !}, \text { if } n \text { is a positive integer }
$$

\section{Hence}

$$
F^{(x)}(0)=\left[\frac{d^{n}}{d z_{1}^{n}} F\left(z_{1}\right)\right]_{z_{1}=0}
$$

$$
\frac{1}{\pi} \int_{-\pi}^{\pi} F\left(z_{1}\right) d \phi_{1}=2 F(0)
$$




$$
\left.\begin{array}{l}
\frac{1}{\pi} \int_{-\pi}^{\pi} \cos n \phi_{1} F\left(z_{1}\right) d \phi_{1}=\frac{\rho_{1}{ }^{n} F^{(n)}(0)}{n !} \\
\frac{1}{\pi} \int_{-\pi}^{\pi} \sin n \phi_{1} F\left(z_{1}\right) d \phi_{1}=\frac{i \rho_{1}{ }^{n} F^{(n)}(0)}{n !}
\end{array}\right\} \text { if } n \text { is a positive integer }
$$

Multiplying 57a by $\rho_{1} d \rho_{1}$, and integrating from $\rho_{1}=0$ to $\rho_{1}=\mathrm{d}_{1} / 2$, gives, on dividing by $d_{1}^{2} / 4$,

$$
\iint \frac{d S_{1}}{S_{1}} F\left(z_{1}\right) d \phi_{1}=F(0)
$$

Similarly, from $57 \mathrm{~b}$ and $57 \mathrm{c}$, we find

$$
\iint \frac{d S_{1}}{S_{1}} \rho_{1} \cos n \phi_{1} F\left(z_{1}\right) d \phi_{1}=\frac{1}{n+3}\left(\frac{d_{1}}{2}\right)^{n+1} \frac{F^{(n)}(0)}{n !}
$$

$\left.\iint \frac{d S_{1}}{S_{1}} \rho_{1} \sin n \phi_{1} F\left(z_{1}\right) d \phi_{1}=\frac{i}{n+3}\left(\frac{d_{1}}{2}\right)^{n+1} \frac{F^{(n)}(0)}{n !}\right\}$, if $n$ is a positive integer.

Taking $F\left(z_{1}\right)=f\left(\frac{z_{2}-z_{1}}{2 \pi p}\right)$, it is evident that the first average over the circle $S_{1}$ is

$$
\bar{f}\left(\frac{z_{2}-z_{1}}{2 \pi p}\right)=f\left(\frac{z_{2}}{2 \pi p}\right)=f\left(\frac{z_{0}+\rho_{2} e^{i \phi_{3}}}{2 \pi p}\right) .
$$

Letting $z_{2}^{\prime}$ denote $\rho_{2} e^{i \phi_{2}}$, the same theorem applies for averaging over the second circle, $S_{2}$. give

Consequently, the four functions in eq 56 , which are twice averaged,

$$
\begin{aligned}
-2 N \overline{\bar{f}}+\overline{\bar{f}_{1}}+\frac{\alpha}{\pi} \overline{\bar{f}_{2}}+\frac{|\alpha|}{\pi} \log \left(\frac{z_{2}-z_{1}}{2 \pi p}\right)= \\
=-2 N f\left(\frac{z_{0}}{2 \pi p}\right)+f_{1}\left(\frac{z_{0}}{2 \pi p}\right)+\frac{\alpha}{\pi} f_{2}\left(\frac{z_{0}}{2 \pi p}\right)+\frac{|\alpha|}{\pi} \log \left(\frac{z_{0}}{2 \pi p}\right) \cdot
\end{aligned}
$$

Similarly, the formulas $58 \mathrm{~b}$ and $58 \mathrm{c}$ suffice for evaluating the last integral in eq 56 .

The eq 56 then becomes

$$
\begin{aligned}
M_{\alpha}=L_{s}+2 \omega_{x}(l) & +\pi D\left\{C_{\alpha}+\frac{2 \alpha}{\pi}\left(\frac{x_{0}}{2 \pi p}\right)\right. \\
+ & \left(\frac{x_{0}}{2 \pi p}\right)\left(\frac{x_{0}+2 p \alpha}{2 \pi p}\right)\left[.85+2 \pi \phi_{1}-2 \log \left(\frac{2 D}{\pi p}\right)\right] \\
+ & \frac{1}{4}\left(\frac{E}{k}-1\right)\left[\left(1+D c_{r_{1}}\right)\left(\frac{d_{1}}{2 \pi p}\right)^{2}+\left(1+D c_{r_{2}}\right)\left(\frac{d_{2}}{2 \pi p}\right)^{2}\right]
\end{aligned}
$$




$$
\begin{gathered}
-2 N f\left(\frac{z_{0}}{2 \pi p}\right)+f_{1}\left(\frac{z_{0}}{2 \pi p}\right)+\frac{\alpha}{\pi} f_{2}\left(\frac{z_{0}}{2 \pi p}\right)+\frac{|\alpha|}{\pi} \log \left(\frac{z_{0}}{2 \pi p}\right) \\
\left.+\frac{l}{16}\left[\left(c_{x_{1}}+i\left(c_{r_{1}}+\frac{1}{2 \pi p}\right)\right)\left(\frac{d_{1}}{2 \pi p}\right)^{2}-\left(c_{x_{2}}+i\left(c_{r_{2}}+\frac{1}{2 \pi p}\right)\right)\left(\frac{d_{2}}{2 \pi p}\right)^{2}\right] f^{\prime}\left(\frac{z_{0}}{2 \pi p}\right)\right\}
\end{gathered}
$$

where the real part is to be taken and

$$
z_{0}=x_{0}+i\left(a_{2}-a_{1}\right)=q e^{i \beta}
$$

Referring to eq 53, we find

$$
\begin{aligned}
& \mathscr{R} f\left(\frac{z_{0}}{2 \pi p}\right)=\mathscr{R} \log \left(2 \sin \frac{z_{0}}{2 p}\right)=\mathscr{R} \log 2 \sin \left[\frac{x_{0}+i\left(a_{2}-a_{1}\right)}{2 p}\right] \\
& =\frac{1}{2} \log 2\left[\cosh \left(\frac{a_{2}-a_{1}}{p}\right)-\cos \frac{x_{0}}{p}\right] \\
& f^{\prime}\left(\frac{z_{0}}{2 \pi p}\right)=\pi \cot \frac{z_{0}}{2 p}=\frac{\pi\left[\sin \frac{x_{0}}{p}-i \sinh \left(\frac{a_{2}-a_{1}}{p}\right)\right]}{\cosh \left(\frac{a_{2}-a_{1}}{p}\right)-\cos \frac{x_{0}}{p}} \\
& \mathscr{R} f_{1}\left(\frac{z_{0}}{2 \pi p}\right)=-2 \sum_{s=2}^{\infty} \frac{\zeta(2 s-1)}{s}\left(\frac{q}{2 \pi p}\right)^{2 s} \cos 2 s \beta \\
& \mathscr{R} f_{2}\left(\frac{z_{0}}{2 \pi p}\right)=-2 \sum_{s=2}^{\infty} \frac{\zeta(2 s-1)}{2 s-1}\left(\frac{q}{2 \pi p}\right)^{2 s-1} \cos (2 s-1) \beta=\frac{2 \gamma x_{0}}{2 \pi p} \\
& +\log \left|\frac{\Gamma\left(1+\frac{z_{0}}{2 \pi p}\right)}{\Gamma\left(1-\frac{z_{0}}{2 \pi p}\right)}\right|
\end{aligned}
$$

Using these real values in eq 59 gives the general formula for $M_{\alpha}$ when the wires are circular. For practical purposes, this may be simplified, since any single-layer, bifilar winding which is to be used as an absolute standard of mutual inductance could be given the following characteristics.

A. The two wires have practically the same diameter and the same treatment, so that the terms of eq 59 which contain the factor $f^{\prime}$ will cancel each other $\left(c_{x_{1}}=c_{x_{2}}\right.$ and $\left.c_{\tau_{1}}=c_{\tau_{2}}\right)$.

B. The mean radii $a_{1}$ and $a_{2}$ are approximately equal, so that we may place $z_{0}=x_{0}$ in all except the principal correction term of first order, which is $-2 N f\left(z_{0} / 2 \pi \rho\right)$.

C. The helical windings are nearly equally spaced, so that $z_{0}=x_{0}=\pi p$ in all terms except the principal correction term. In this case, by eq $59 \mathrm{~d}$, 


$$
f_{2}\left(\frac{z_{0}}{2 \pi p}\right)=f_{2}\left(\frac{1}{2}\right)=\gamma+\log \frac{\Gamma\left(1+\frac{1}{2}\right)}{\Gamma\left(1-\frac{1}{2}\right)}=\gamma-\log 2=-.116
$$

$\log \frac{z_{0}}{2 \pi p}=-\log 2=-.693$, and from $=53 f$

$$
\begin{aligned}
& { }_{1}\left(\frac{z_{0}}{2 \pi p}\right)=f_{1}\left(\frac{1}{2}\right)=-2 \sum_{s=2}^{\infty} \frac{\zeta(2 s-1)}{s 4^{s}}=-2\left[\sum_{s=2}^{\infty} \frac{1}{s 4^{s}}+\sum_{s=2}^{\infty}\left(\frac{\zeta(2 s-1)-1}{s 4^{s}}\right)\right] \\
& =\frac{1}{2}+2 \log \frac{3}{4}-2 \sum_{s=2}^{\infty}\left[\frac{\zeta(2 s-1)-1}{s 4^{s}}\right]=-.087
\end{aligned}
$$

The eq 59 then becomes

$$
\begin{aligned}
M_{\alpha}=L_{s} & +2 \omega_{x}(l)+\pi D\left\{C_{\alpha}+.12+1.31 \frac{\alpha}{\pi}-.693 \frac{|\alpha|}{\pi}\right. \\
+\left(\frac{1}{2}+\frac{\alpha}{\pi}\right)\left(\pi \phi_{1}-\log \frac{2 D}{\pi p}\right)- & N \log 2\left(\cosh \frac{a_{2}-a_{1}}{p}-\cos \frac{x_{0}}{p}\right) \\
+ & \left.\frac{1}{2}\left(\frac{E}{k}-1\right)\left(1+D c_{r}\right)\left(\begin{array}{c}
d \\
2 \pi p
\end{array}\right)^{2}\right\}
\end{aligned}
$$

It may be noticed that the term $1+D c_{\tau}$ is +1 or -1 , according as the current distribution is uniform or "natural." The approximate value of the principal correction term within the brace is $-N \log$ $4=-1.38629 \mathrm{~N}$.

When the initial sections of both wires are in the same axial plane, $\alpha=0$, the special case of eq 60 then is

$$
\begin{aligned}
M_{0}= & L_{s}+2 \omega_{x}(l)-\pi D\left\{-.12+\frac{1}{6} \log \left(\frac{2 D}{\pi p}\right)-\frac{\pi \phi_{1}}{6}+\frac{k^{\prime}}{2 k}\left(1-\frac{k^{\prime}}{k} \sin ^{-1} k\right)\right. \\
& \left.-\frac{1}{2}\left(\frac{E}{k}-1\right)\left(1+D c_{r}\right)\left(\frac{d}{2 \pi p}\right)^{2}+N \log 2\left(\cosh \frac{a_{2}-a_{1}}{p}-\cos \frac{x_{0}}{p}\right)\right\} .
\end{aligned}
$$

When $\alpha=-\pi$, the special case of eq 60 is

$$
\begin{aligned}
M_{-\pi} & =L_{s}+2 \omega_{x}(l)-\pi D\left\{\frac{1}{6} \log \left(\frac{2 D}{\pi p}\right)+\frac{\pi \phi_{1}}{3}+\frac{1}{2 k}-\frac{1}{4 k^{2}} \log \left(\frac{1+k}{1-k}\right)\right. \\
& \left.-\frac{1}{2}\left(\frac{E}{k}-1\right)\left(1+D c_{r}\right)\left(\begin{array}{c}
d \\
2 \pi p
\end{array}\right)^{2}+N \log 2\left(\cosh \frac{a_{2}-a_{1}}{p}-\cos \frac{x_{0}}{p}\right)\right\} \cdot(60 \mathrm{~b})
\end{aligned}
$$

In this equation the lead wires may be taken in the same axial plane but on opposite sides of the cylindrical form, as shown in figure 2 . As a guide in computing, it may be useful to remember that terms smaller than $D / 4$ have been neglected in the derivation of formula 60 . This corresponds to an uncertainty of about 0.1 in the value of all the terms within the brace which has the factor $\pi D$. 
For the sake of comparison, the self inductance, $L$, of either of the two equal helical wires (paper 3 ) may be put in the form

$L=L_{s}+2 \omega_{x}(l)$

$$
\begin{aligned}
+\pi D & \left\{2 N\left[\log \frac{2 \pi p}{D}-89473\right]+\frac{1}{3} \log \frac{D}{2 p}\right. \\
+ & \frac{1}{2}\left(\frac{E}{k}-1\right)\left(1+D c_{r}\right)\left(\frac{d}{2 \pi p}\right)^{2} \\
& \left.-\frac{\pi \phi_{1}}{3}-\frac{k^{\prime}}{2 k}\left(1-\frac{k^{\prime}}{k} \sin ^{-1} k\right)\right\},
\end{aligned}
$$

where $\omega_{x}(l)$ in this equation and in eq $55,56,60 \mathrm{a}$ and $60 \mathrm{~b}$ is computed by eq 22 .

\section{EFFECT OF LEAD WIRES}

When the lead wires are each parallel and close to a generator of the cylindrical form, it is simpler to use, in eq 60 , instead of the term $2 \omega_{x}(l)$, the term $2 \bar{\omega}_{x}(l, \alpha)$, which takes account of all axial currents in lead wires and helices together. In paper 1 eq 42 to 46 are given the relations which in the case $r_{1}=r_{2}=D / 2$ are equivalent to

$$
\begin{aligned}
2 \bar{\omega}_{x}(l, \alpha)=2\left\{\frac{2 D}{\pi}\left(\frac{E}{k}-1\right)+R(\alpha)-\sqrt{l^{2}+R_{(\alpha)}^{2}}\right. \\
\left.\frac{-l^{2}}{\sqrt{l^{2}+D^{2}}} \log 2+l \cdot \log \frac{D\left[l+\sqrt{\left.l^{2}+R_{(\alpha)}^{2}\right]}\right.}{R(\alpha)\left[l+\sqrt{\left.l^{2}+D^{2}\right]}\right.}\right\}
\end{aligned}
$$

where

$$
R^{2}(\alpha)=r_{l_{1}}^{2}-2 r_{l_{1}} r_{l_{2}} \cos \alpha+r_{1_{2}}^{2}
$$

the distance of the lead wires from the axis of the cylindrical form being $r_{l_{1}}$ and $r_{l_{2}}$, where $r_{l_{1}}-a$ and $r_{l_{2}}-a$ are small, so that when $\alpha= \pm \pi, R( \pm \pi)$ is practically the same as the mean diameter, $D$, of the coils.

The term $2 \varpi_{x}(l, \alpha)$ is to be substituted for (not added to) the term $2 \omega_{x}(l)$ in eq 60 and its special cases.

\section{MODIFICATION REQUIRED WHEN THE REFERENCE SECTIONS ARE NOT QUITE CIRCULAR}

In this case, we first find the position of the centroids of the sections, thus determining $a_{1}, a_{2}$, and $x_{0}$, so that the points $0_{1}$ and $0_{2}$ are determined. With these points as centers, we describe two circles whose diameters, $d_{1}$ and $d_{2}$ are determined by

$$
\pi\left(\frac{d_{1}}{2}\right)^{2}=S_{1} \text { and } \pi\left(\frac{d_{2}}{2}\right)^{2}=S_{2}
$$

The equations of the boundary curves of $S_{1}$ and $S_{2}$ may then be put in the form

$$
\rho_{1}=\frac{d_{1}}{2}\left[1+\epsilon_{1}\left(\phi_{1}\right)\right] \text { and } \rho_{2}=\frac{d_{2}}{2}\left[1+\epsilon_{2}\left(\phi_{2}\right)\right],
$$

where $\epsilon_{1}$ and $\epsilon_{2}$ are functions of $\phi_{1}$ and $\phi_{1}$, which must take on positive and negative values. We assume that the order of magnitude of $\left|\epsilon_{1}\right|$ and $\left|\epsilon_{2}\right|$ is not greater than that of $p / a$. 
We may at first consider $S_{2}$ to be exactly circular. The section $S_{1}$ differs from the area of a circle of diameter $d_{1}$ by the area of a narrow strip whose radial breadth is

$$
\frac{d_{1}}{2} \epsilon_{1}\left(\phi_{1}\right)
$$

Since the centroid of circle is at $O_{1}$, and also the centroid of $S_{1}$ is at $O_{1}$, it follows that the centroid of this narrow strip is at $O_{1}$. Hence we have imposed four conditions upon the small function $\epsilon_{1}\left(\phi_{1}\right)$.

Since the element of area of the strip is $\left(\frac{d_{1}}{2}\right)^{2} \epsilon_{1}\left(\phi_{1}\right) d \phi_{1}$, these four conditions are

$\left|\epsilon_{1}\left(\phi_{1}\right)\right| \leqslant C \frac{p}{a}$, (where $C$ is of the order of magnitude of unity)

$\int_{-\pi}^{\pi} \epsilon_{1}\left(\phi_{1}\right) d \phi_{1}=0$

$\int_{-\pi}^{\pi} \epsilon_{1}\left(\phi_{1}\right) \cos \phi_{1} d \phi_{1}=0$

$\int_{-\pi}^{\pi} \epsilon_{1}\left(\phi_{1}\right) \sin \phi_{1} d \phi_{1}=0$

The eq $65 \mathrm{~b}$ states that $d_{1}$ has been so chosen that the area of the noncircular section, $S_{1}$, is $\pi\left(\frac{d_{1}}{2}\right)^{2}$

Equations $65 \mathrm{c}$ and $65 \mathrm{~d}$ state that $O_{1}$ is the centroid of the section $S_{1}$ and also of the circle.

The function $\epsilon_{1}$ must be a periodic function with period $2 \pi$. Hence it is developable in a Fourier's series in the form

$$
\epsilon_{1}\left(\phi_{1}\right)=\sum_{n=2}^{\infty}\left(C_{n} \cos n \phi_{1}+D_{n} \sin n \phi_{1}\right),
$$

where the term $n=1$ must be absent because of eq $65 \mathrm{c}$ and $65 \mathrm{~d}$. The constant term is absent because of condition $65 \mathrm{~b}$.

As explained in the derivation of eq 59, the only effect of this deviation of $S_{1}$ from exact circularity will consist in a correction (of second order) that must be made to the principal correction term of eq 59 , which is the term $-2 N f\left(z_{0} / 2 \pi p\right)$ within the brace.

Instead of the term $f\left(z_{0} / 2 \pi p\right)$ in eq 59 , we must place

$$
\iint \frac{d S_{1}}{S_{1}} \iint \frac{d S_{2}}{S_{2}} f\left(\frac{z_{2}-z_{1}}{2 \pi p}\right)=\iint \frac{d S_{1}}{S_{1}} f\left(\frac{z_{0}-z_{1}}{2 \pi p}\right),
$$

where the integral is now taken over the section $S_{1}$, whose boundary curve is given by eq $64 \mathrm{~b}$. When this same integral was taken over the circular section, its value was found to be $f\left(z_{0} / 2 \pi p\right)$. Hence the correction term to be added to eq 59 is equal to $1 / S_{1}$ times the integral of $\left.f\left(z_{0}-z_{1}\right) / 2 \pi p\right)$ taken over the area of the narrow strip. This gives, since $\epsilon_{1}$ is small, the correction

$$
\Delta_{1}=\frac{1}{\pi} \int_{-\pi}^{\pi} \epsilon_{1}\left(\phi_{1}\right) f\left(\frac{z_{0}-z_{1}}{2 \pi p}\right) d \phi_{1}
$$


where the integral is taken around the circle $\rho_{1}=d_{1} / 2$. When $\Delta_{1}$ is found, the term $-2 N f\left(z_{0} / 2 \pi p\right)$ in eq 59 must be replaced by $-2 N\left[\Delta_{1}+f\left(\frac{z_{0}}{2 \pi p}\right)\right]$. This will make a change in the formula 59 by an amount which cannot be larger than a second-order term.

If, in eq 67, we insert the series 66 and make use of eq $57 \mathrm{~b}$ and $57 \mathrm{c}$, we find

Similarly,

$$
\Delta_{1}=\sum_{n=2}^{\infty} \frac{(-1)^{n}\left(C_{n}+i D_{n}\right)}{n !}\left(\frac{d_{1}}{4 \pi p}\right)^{n} f^{(n)}\left(\frac{z_{0}}{2 \pi p}\right)
$$

$$
\Delta_{2}=\sum_{n=2}^{\infty} \frac{(-1)^{n}\left(C_{n}^{\prime}+c D\right)_{n}^{\prime}}{n !^{\prime}}\left(\frac{d_{2}}{4 \pi p}\right)^{n} f^{(n)}\left(\frac{z_{0}}{2 \pi p}\right)
$$

when the boundary curve of $S_{2}$ is of the type of eq $64 \mathrm{~b}$ and

$$
\epsilon_{2}\left(\phi_{2}\right)=\sum_{n=2}^{\infty}\left(C_{n}^{\prime} \cos n \phi_{2}+D_{n}^{\prime} \sin n \phi_{2}\right) .
$$

Therefore, the correction is made to eq $59,60,60 \mathrm{a}$, and $60 \mathrm{~b}$ by adding within the brace which is multiplied by $\pi D$, the term $-2 N\left(\Delta_{1}+\Delta_{2}\right)$.

Perhaps the case of most practical interest is the elliptic section

$$
\frac{x_{1}^{0^{2}}}{A_{1}^{2}}+\frac{\left(r_{1}-a_{1}\right)^{2}}{B_{1}^{2}}=1
$$

or in polar coordinates

$$
\rho_{1}=\frac{A_{1} B_{1}}{\sqrt{\frac{A_{1}^{2}+B_{1}^{2}}{2}-\left(\frac{A_{1}^{2}-B_{1}^{2}}{2}\right) \cos 2 \phi_{1}}} .
$$

If $\left(A_{1}-B_{1}\right) / A_{1}$ is small, this becomes, neglecting $\left(A-B_{1}\right)^{2} / A^{2}$,

$$
\rho_{1}=\sqrt{A_{1} B_{1}}\left[1+\frac{A_{1}-B_{1}}{2 A_{1}} \cos 2 \phi_{1}\right] .
$$

The diameter, $d_{1}$, of a circle whose area is equal to the area $\pi A_{1} B_{1}$ of the ellipse is given by $d_{1} / 2=\sqrt{A_{1} B}$, hence the boundary curve is

$$
\rho_{1}=\frac{d_{1}}{2}\left[1+\frac{A_{1}-B_{1}}{d_{1}} \cos 2 \phi_{1}\right],
$$

so that, on reference to eq $64 \mathrm{~b}$, we find

$$
\epsilon_{1}\left(\phi_{1}\right)=\frac{A_{1}-B_{1}}{d_{1}} \cos 2 \phi_{1} .
$$

The only Fourier's coefficient in eq 66 which is not zero is $C_{2}$, which is equal to $\left(A_{1}-B_{1}\right) / d_{1}$. Hence, by eq $68 \mathrm{a}$, 


$$
\Delta_{1}=\frac{1}{2}\left(\frac{A_{1}-B_{1}}{d_{1}}\right)\left(\frac{d_{1}}{4 \pi p}\right)^{2} f^{(2)}\left(\frac{z_{0}}{2 \pi p}\right)
$$

From eq $53 \mathrm{j}$, we find $\ldots f^{(2)}\left(\frac{z_{0}}{2 \pi p}\right)=-\frac{\pi^{2}}{\sin ^{2} \frac{z_{0}}{2 p}} \cdot$

Taking the real part

$$
R f^{(2)}\left(\frac{z_{0}}{2 \pi p}\right)=-\pi^{2} \frac{\cosh \left(\frac{a_{2}-a_{1}}{2 p}\right)-\cos \frac{x_{0}}{2 p}}{\cosh \left(\frac{a_{2}-a_{1}}{2 p}\right)+\cos \left(\frac{x_{0}}{2 p}\right)},
$$

so that

$$
\Delta_{1}=-\frac{1}{2}\left(\frac{A_{1}-B_{1}}{d_{1}}\right)\left(\frac{d_{1}}{2 p}\right)^{2} \frac{\cosh \left(\frac{a_{2}-a_{1}}{2 p}\right)-\cos \frac{x_{0}}{2 p}}{\cosh \frac{a_{2}-a_{1}}{2 p}+\cos \frac{x_{0}}{2 p}}
$$

The correction, $\Delta_{2}$, by change of subscripts, takes account of the fact that the reference section of the second wire is an ellipse, as in eq 69, with semiaxes $A_{2}$ and $B_{2}$. In these equations $A_{1}$ may be greater or less than $B_{1}$. Hence with both wires of elliptic sections the correction to be added to eq 59 within the brace is the real quantity

$$
\begin{aligned}
& -2 N\left(\Delta_{1}+\Delta_{2}\right) \\
& =N\left[\left(\frac{A_{1}-B_{1}}{\alpha_{1}}\right)\left(\frac{d_{1}}{2 p}\right)^{2}+\left(\frac{A_{2}-B_{2}}{d_{2}}\right)\left(\frac{d_{2}}{2 p}\right)^{2}\right]_{\cosh \frac{a_{2}-a_{1}}{2 p}+\cos \frac{x_{0}}{2 p}}^{\cosh \frac{a_{2}-a_{1}}{2 p}-\cos \frac{x_{0}}{2 p}} .
\end{aligned}
$$

Since, by hypothesis, the order of magnitude of $\left(A_{1}-B_{1}\right) / d_{1}$, etc., is not greater than that of $p / a$, it is evident that the correction term 71 is not greater than second order. If $\left(A-{ }_{1} B_{1}\right) / d_{1}$ is of the order of magnitude of $p^{2} / a^{2}$, the correction for ellipticity is entirely negligible in the formulas 59 to $60 \mathrm{~b}$.

In the eq 60 and its special cases $60 \mathrm{a}$ and $60 \mathrm{~b}$ we have placed $x_{0}=\pi p$ and $a_{2}-a_{1}=0$ in the second-order terms of eq 59. Hence for the eq $60,60 \mathrm{a}$, and $60 \mathrm{~b}$, the correction term 71 reduces to

$$
-2 N\left(\Delta_{1}+\Delta_{2}\right)=N\left[\left(\frac{A_{1}-B_{1}}{d_{1}}\right)\left(\frac{d_{1}}{2 p}\right)^{2}+\left(\frac{A_{2}-B_{2}}{d_{2}}\right)\left(\frac{d_{2}}{2 p}\right)^{2}\right] \text {. }
$$

We have idealized the actual helical wire, $w_{1}$, as one which is cut in a circular section by a plane through the axis of the cylindrical form. This is the circle shown in the reference plane, or $x y$ plane, of figure 1. However, a more natural view of the wire is one which is cut in a circular section by a plane normal to its central filament. The reference section of such a wire would be slightly elliptical. If its semiaxes are $A_{1}$ and $B_{1}$, it will be shown that the ratio $\left(A_{1}-B_{1}\right) / d_{1}$ is equal to $p^{2} / 2 a^{2}$, so that by eq 71 the correction is of third order and is therefore not to be made to the formula here given, since we have neglected an unknown number of terms of third order. 
To examine this second view of the helical wire, consider the three eq 1 , which may be written (dropping subscripts)

$\left.\begin{array}{l}x=x^{0}+p \theta \\ y=r \cos \theta \\ z=r \sin \theta\end{array}\right\}$, where $\left\{\begin{array}{c}-2 \pi p<x^{0}<2 \pi p \\ -\infty<\theta<\infty \\ 0<r<\infty\end{array}\right\}$.

Since the assignment of the values of $x^{0}, r$, and $\theta$ uniquely determines the position of a point in space, they constitute a system of space coordinates which are not orthogonal. With this specification of their ranges, they are uniquely determined when $x, y$, and $z$ are given. Any equation of the form $x^{0}=\phi(r, \theta)$ represents a surface. In particular, the equation independent of $\theta$

$x^{0}=\phi(r)$

represents the surface of a helicoidal wire. This surface cuts the cylinder $r=$ constant in a helix whose equations would be given by eq 72 on replacing $x^{0}$ by $\phi(r)$, and holding $r$ constant, while $\theta$ is varied.

The curve in which the surface of eq 73 is cut by a plane, $\theta=c=$ constant, is congruous with the curve in which the surface is cut by any other plane, $\theta=c_{1}=$ constant. The two curves may be made to coincide by giving one a translation in the $x$ direction, followed by a rotation about the $x$ axis.

Taking as a representative cutting plane, the plane $\theta=0$ gives a curve whose rectangular equations are by eq 72

$x=\phi(y)$

$z=0$.

Hence if we have the equation of the surface of any helical wire in the form of eq 73, we may find the curve bounding its reference section of figure 1 by replacing $x^{0}$ by $x$ and $r$ by $y$ in eq 73 .

Next, suppose the equations of any closed space curve are given in rectangular coordinates in the form

$f_{1}(x, y, z)=0$

$f_{2}(x, y, z)=0$.

Let it be required to find the equation of the surface of a helical wire which passes through this assigned curve.

Writing the equations of the given curve in helicoidal coordinates $x^{0}, r$, and $\theta$, they are, by eq 72 ,

$r_{1}\left(x^{0}+p \theta, r \cos \theta, r \sin \theta\right)=0$

$f_{2}\left(x^{0}+p \theta, r \cos \theta, r \sin \theta\right)=0$.

Eliminating $\theta$ between these two equations, we obtain a relation between $x^{0}$ and $r$, which, if solved for $x^{0}$, takes the form of eq 73. It is therefore the required equation of the surface of the helicoidal wire but expressed in helicoidal coordinates. Its reference section is found by placing $x^{0}=x, r=y$, as in eq 74 .

Such elimination of $\theta$ is seldom possible, so that the reference section may be considered as a curve defined in parametric form 
by placing $x^{0}=x$ and $r=y$ in the two eq $76 \mathrm{a}$ and $76 \mathrm{~b}$. The variable line parameter is $\theta$.

Consider next another rectangular system of coordinates obtained by rotating the $x, y, z$ system of figure 1 about the $y$ axis, through a positive angle, $\gamma$. Any point, $P$, in space has the rectangular coordinates $x, y, z$, in the old system and $x^{\prime}, y^{\prime}, z^{\prime}$, in the new, where

$x^{\prime}=x \cos \gamma-z \sin \gamma$

$y^{\prime}=y$

$z^{\prime}=x \sin \gamma+z \cos \gamma$.

Suppose that we desire the equation of the surface of a helical wire whose section by the plane $z^{\prime}=0$ is a circle of diameter $d$ with center at $x^{\prime}=0, y^{\prime}=a, z^{\prime}=0$. The equations of this circle (as a space curve) are

$x^{\prime 2}+\left(y^{\prime}-a\right)^{2}=\frac{d^{2}}{4}$

$z^{\prime}=0$

or by eq 77

$$
\begin{gathered}
(x \cos \gamma-z \sin \gamma)^{2}+(y-a)^{2}=\frac{d^{2}}{4} \\
x \sin \gamma+z \cos \gamma=0 .
\end{gathered}
$$

The two latter correspond to eq $75 \mathrm{a}$ and $75 \mathrm{~b}$. In helicoidal coordinates, these become

$$
\begin{gathered}
{\left[\left(x^{\circ}+p \theta\right) \cos \gamma-r \sin \theta \sin \gamma\right]^{2}+(r \cos \theta-a)^{2}-\frac{d^{2}}{4}=0} \\
\left(x^{\circ}+p \theta\right) \sin \gamma+r \sin \theta \cos \gamma=0,
\end{gathered}
$$

which are special cases of the general eq $76 \mathrm{a}$ and $76 \mathrm{~b}$. The relation between $x^{0}$ and $r$ resulting from the elimination of $\theta$ between $80 \mathrm{a}$ and $80 \mathrm{~b}$ would be the equation of the surface of a helicoidal wire which is cut by the plane $z^{\prime}=0$ (i. e. $z=-x \operatorname{tau} \gamma$ ) in the circular section defined by $78 \mathrm{a}$ and $78 \mathrm{~b}$. Instead of attempting to eliminate $\theta$, it is better to retain it as a parameter. The equations of the boundary curve of the reference section of this wire is found, as explained above, by replacing $x^{0}$ by $x$ and $r$ by $y$. They are

$$
\begin{gathered}
{[(x+p \theta) \cos \gamma-y \sin \theta \sin \gamma]^{2}+(y \cos \theta-a)^{2}-\frac{d^{2}}{4}=0} \\
(x+p \theta) \sin \gamma+y \sin \theta \cos \gamma=0
\end{gathered}
$$

(together with $z=0$ ).

The second view of the wire corresponds to

$$
\cos \gamma=\frac{a}{\sqrt{a^{2}+p^{2}}} \text { and } \sin \gamma=\frac{p}{\sqrt{a^{2}+p^{2}}},
$$


which, as shown by 72 , makes the plane $z^{\prime}=0$ normal to the central filament of the wire at $x=0, y=a, z=0$. Using eq 82 , eq 81 may be written

$$
\begin{gathered}
x=-p \theta-y\left(\frac{a \sin \theta}{p}\right) \\
\left(a^{2} \sin ^{2} \theta+p^{2}\right) y^{2}-2 p^{2} a \cos \theta \cdot y+p^{2}\left(a^{2}-\frac{d^{2}}{4}\right)=0,
\end{gathered}
$$

which are the parametric equations of the boundary curve of the reference section of the wire. The last was obtained by eliminating $x$ between 81a and 81b. On solving this equation for $y$, we obtain the following parametric equations for the boundary of the reference section of this wire:

$$
\begin{gathered}
x=-p \theta-y\left(\frac{a \sin \theta}{p}\right) \\
y=\frac{1}{1+\left(\frac{a \sin \theta}{p}\right)^{2}}\left[a \cos \theta \pm \sqrt{\rho^{2}-\left(a^{2}+p^{2}-\rho^{2}\right)\left(\frac{a \sin \theta}{p}\right)^{2}}\right]
\end{gathered}
$$

where $\rho=d / 2$.

From this we find

$$
\frac{d y}{d x}=-\frac{a y \sin \theta}{p(a \cos \theta-y)}
$$

The section is a closed curve symmetrical with respect to the line $x=0$.

The real values of $y$ correspond to values of the parameter $\theta$ in the range $-\theta_{0}<\theta<\theta_{0}$,

where

$$
\theta_{0}=\sin ^{-1} \frac{p \rho}{a} \frac{p}{a^{2}+p^{2}-\rho^{2}}
$$

If we now introduce the facts that $p / a$ and $\rho / a(=d / 2 a)$ are small, it is found that

$$
\theta_{0}=\frac{p d}{2 a^{2}}\left[1+\operatorname{Zero}\left(\frac{p^{2}}{a^{2}}\right)\right]
$$

Hence in eqs $83 \mathrm{a}$ and $83 \mathrm{~b}$ we may expand $\sin \theta$ and $\cos \theta$ in powers of $\theta$ This gives, neglecting terms smaller than $p^{2} / a^{2}$,

$$
\begin{gathered}
x=-\frac{a \theta}{p}\left(1+\frac{p^{2}}{a^{2}}\right) y \\
(y-a)^{2}+\left(\begin{array}{c}
a \theta \\
p
\end{array}\right)^{2} y^{2}=\frac{d^{2}}{4} .
\end{gathered}
$$

On eliminating $\theta$ between these equations, we find for the boundary of the reference section, the equation 


$$
\frac{x^{2}}{A^{2}}+\frac{(y-a)^{2}}{B^{2}}=1
$$

where

so that

$$
A=\frac{d}{2}\left(1+\frac{p^{2}}{a^{2}}\right) \text { and } B=\frac{d}{2}
$$

$$
\frac{A-B}{d}=\frac{p^{2}}{2 a^{2}}
$$

as stated above.

Since terms smaller than second order have been neglected, the formula here obtained for the mutual inductance of the two helical wires has the same validity for either of the two modes of defining the helical wire. This applies to formulas for mutual inductance and selfinductance obtained in the earlier papers quoted above. The conclusion is important because the absolute determinations of the ohm and of the ampere which have been made at this Bureau during the last decade are based upon the use of these formulas, in which the terms of second order have not been negligible. However, if the wire is found to have an elliptic section whose ellipticity is a small quantity of the first order, then the correction 71 must be applied.

\section{SUMMARY}

The formula for the mutual inductance of the two helical wires of wire diameter $d$, which are wound bifilarly in a single layer on a circular cylindrical form is given by eq 60 . In this formula the azimuthal angle, $\alpha$ between the initial ends of wires $w_{1}$ and $w_{2}$ lies between $-\pi$ and $\pi$, inclusive. When $\alpha=-\pi$, the central filament of wire $w_{2}$ begins at $0_{2}^{\prime}$ in figure 2 ; when $\alpha=0$, it begins at $0_{2} ;$ and when $\alpha=+\pi$, at $0_{2}^{\prime \prime}$; the central filament of wire $w_{1}$ beginning at $0_{1}$ in all cases. The total number of turns in each helix is $N$. The cylindrical radii, $a_{1}$ and $a_{2}$, of the central filaments are considered so nearly equal that $a_{2}-a_{1} / 2 \pi p$ is small where $2 \pi p$ is the pitch of the windings, the axial length, $l$, being $2 \pi p N$. The principal term of eq 60 is $L_{s}$, which represents the self inductance of a circular cylindrical current sheet of length $l$ and radius $a=\left(a_{1}+a_{2}\right) / 2=D / 2$. This sheet has angular component of current only.

The term $2 \omega_{x}(l)$ in eq 60 represents the effect of axial components of current in the helices. It is a relatively small term of second order which may be computed by eq 22 , but which should be replaced by $2 \bar{\omega}_{x}(l)$ given by eq 62 when the lead wires are close to a generator of the cylinder, as this takes into account the effect of axial currents in lead wires and helices together.

The principal correction term in eq 60 contains the large factor, $N$. In this term, $x_{0}$ is the (positive) axial component of the line from $0_{1}$ to $0_{2}$ in figure 1 . In all other correction terms, $x_{0}$ has been placed equal to $\pi p$ and $a_{2}-a_{1}$ equal to zero, but allowance is made in this term for the possibility that measurements will show slight deviations from these values. The value $x_{0}=\pi p$ corresponds to the fact that the windings of one wire are equally spaced between those of the other.

In section VI, a modification of the formula is given to apply when the reference sections of the wires are only approximately circular. 


\section{LIST OF SYMBOLS}

$a_{1}=$ mean radius of primary wire, $w_{1}$.

$a_{2}=$ mean radius of secondary wire, $w_{2}$.

$d_{1}=$ diameter of wire $w_{1}$.

$d_{2}=$ diameter of wire $w_{2}$.

$2 \pi p=$ the pitch of each winding.

$N=$ number of turns in each winding.

$l=2 \pi p N=$ the axial length of each winding.

$x_{0}=x_{0}^{0}$ of figure 1 =axial distance from center of wire $w_{1}$ to center of wire $w_{2}$, both centers lying in a plane through the $x$ axis. When windings of one are midway between those of the other, $x_{0}=\pi p$. It is assumed that $x_{0}$ is near $\pi p . \quad q$ and $\beta$ defined by $z_{0} \equiv x_{0}+i\left(a_{2}-a_{1}\right) \equiv q e^{i \beta}$, where $-\pi / 2<\beta<\pi / 2$.

$\alpha=$ the difference in azimuth of the initial points of wires $w_{1}$ and $w_{2}$, both wires having the same end planes when $\alpha=-\pi$. It is assumed that $|\alpha|<\pi$.

$c_{x_{1}}, c_{r_{1}}, c_{x_{2}}, c_{r_{2}}=$ partial derivatives of the logarithm of the magnitude of the current density, for points on the respective central filaments (eq 5b). For uniform current distribution they are zero; for the natural distribution

$c_{x_{1}}=c_{x_{2}}=0$ and $c_{r_{1}}=-1 / a_{1}, c_{r_{2}}-1 / a_{2}$.

$D=2 a=a_{1}+a_{2}$.

$k^{2}=\frac{D^{2}}{l^{2}+D^{2}}$ and $k^{\prime 2}=1-k^{2}$.

$K$ and $E$ are the complete elliptic integrals with modulus $k$.

$L_{s}=$ the self inductance of a current sheet of length $l$ and diameter $D$ (eq 16).

$M=$ the mutual inductance of the helical wires, $w_{1}$ and $w_{2}$.

$$
\begin{aligned}
& \zeta(2 s-1)=\sum_{n=1}^{\infty} \frac{1}{n^{2 s-1}}(=45) . \\
& \pi \phi_{1}=\frac{2}{k}[K-E]-k K(=8) .
\end{aligned}
$$

$C_{\alpha}$ defined in eq $53 \mathrm{~d}$.

Conditions $A, B$, and $C$ preceding eq 60 give the assumptions by which eq 60 becomes a special case of eq 59 .

The term $2 \omega_{x}(l)$ in eq $59,60,60 \mathrm{a}$, and $60 \mathrm{~b}$, may be computed by eq 22 and 23. It represents the effect of the axial components of current in the two helical wires, $w_{1}$ and $w_{2}$. The term $2 \bar{\omega}_{x}(l, \alpha)$ may be computed by eq 62 and 63 . When the lead wires are close to the cylindrical form, as shown in figure 2 , the term $2 \bar{\omega}_{x}(l, \alpha)$ should replace $2 \omega_{x}(l)$ in eq 59 to 60 to take account of all axial components of current in helices and lead wires together.

The "helical wire" is assumed to be built up of helical filaments (all with the same pitch) in such a manner that any plane through the $x$ axis cuts the wire in circular sections.

Washington, December 19, 1939. 